\title{
Eine nachhaltige Sicherheitskultur als Transformationsansatz für Industrie 4.0 in kleinen und mittleren Unternehmen
}

\author{
Claas Digmayer, Eva-Maria Jakobs, Anna Borg, \\ Achim Buschmeyer, Cornelia Hahn, Johanna Kluge, \\ Jonathan Reinartz, Jan Westerbarkey und Martina Ziefle
}

\section{Zusammenfassung}

Dieser Beitrag beschreibt einen Transformationsansatz, der kleinere und mittlere Unternehmen (KMU) auf dem Weg zu Industrie 4.0 bei der Entwicklung einer digitalen Sicherheitskultur durch ein strukturiertes Vorgehensmodell sowie geeignete Strategien (Anforderungs-Analyse, Ist-Analyse, Identifikation von Nutzenpotentialen, Entwicklung

C. Digmayer $(\bowtie) \cdot$ E.-M. Jakobs

Textlinguistik und Technikkommunikation, Human-Computer Interaction Center, RWTH Aachen University, Aachen, Deutschland

E-Mail: c.digmayer@tk.rwth-aachen.de; e.m.jakobs@tk.rwth-aachen.de

A. Borg

CBM Gesellschaft für Consulting Business und Management mbH, Bexbach, Deutschland

E-Mail: borg@cbm-ac.de

A. Buschmeyer · C. Hahn

DERICHS u KONERTZ GmbH u Co KG, Aachen, Deutschland

E-Mail: a.buschmeyer@derichsukonertz.de; c.hahn@ derichsukonertz.de

J. Kluge $\cdot$ M. Ziefle

Communication Science, Human-Computer Interaction Center, RWTH Aachen University,

Aachen, Deutschland

E-Mail:kluge@comm.rwth-aachen.de; ziefle@humtec.rwth-aachen.de

J. Reinartz

Forschungsinstitut für Rationalisierung an der RWTH Aachen University, Aachen, Deutschland

E-Mail: Jonathan.Reinartz@ fir.rwth-aachen.de

J. Westerbarkey

Westaflex GmbH, Gütersloh, Deutschland

E-Mail: westerbarkey_jan@westa.net

(C) Der/die Autor(en) 2021

T. Jeske und F. Lennings (Hrsg.), Produktivitätsmanagement 4.0, ifaa-Edition,

https://doi.org/10.1007/978-3-662-61584-3_3 
von Umsetzungsinstrumenten) unterstützt. Der Transformationsansatz erweitert die eher technisch orientierten Herangehensweisen der Arbeitssicherheit, indem ein präventiv und partizipativ erarbeitetes Sicherheitskulturkonzept in den Unternehmen implementiert wird. Dabei werden etablierte Instrumente und Vorgehensmodelle des Arbeits- und Gesundheitsschutzes (AGS) erweitert und an die neuen Anforderungen der digitalisierten Arbeitswelt angepasst. KMU werden so befähigt, neue Anforderungen im AGS durch flexible Lösungen zu bewältigen sowie ein passgenaues Sicherheitskulturkonzept für Industrie $4.0 \mathrm{zu}$ entwickeln und zu etablieren. In diesem Beitrag werden die dazu notwendigen Schritt aufgezeigt und anhand von Praxisbeispielen veranschaulicht. Der Beitrag schließt mit den wichtigsten Befunden (Lessons learned) bei der Etablierung einer Sicherheitskultur für Industrie 4.0.

\subsection{Ausgangssituation}

Ansätze zur Digitalisierung der Wirtschaft fokussieren technische Aspekte. Der vorliegende Beitrag erweitert diese Perspektive durch die Entwicklung eines Transformationsansatzes für die Etablierung eines präventiven und partizipativ erarbeiteten Sicherheitskulturkonzeptes als maßgeblichen Erfolgsfaktor für die Umsetzung von Industrie 4.0 in KMU. Der Ansatz hilft Unternehmen, Ressourcen und Barrieren des Arbeitens in der digitalisierten Welt zu identifizieren, mit den Mitarbeitern ein passgenaues Sicherheitskulturkonzept zu entwickeln und dieses durch konkrete Maßnahmen zu etablieren. Der Ansatz orientiert sich an Zielkriterien wie nachhaltige Wirksamkeit, Flexibilität und die Etablierung einer überbetrieblichen Präventionsallianz auf dem Weg in eine digitalisierte Arbeitswelt. Das Sicherheitskulturkonzept fokussiert Empowerment [28], Respekt und Vertrauen, ethische Standards für Akteure, Freiräume für Risiken sowie die Nutzung impliziten und expliziten Wissens der Mitarbeiter. Der Ansatz geht damit über bisherige Präventionsansätze hinaus. Er nutzt das Potential von Mitarbeitern und Führungskräften und setzt auf höhere Eigenverantwortung bei der Umsetzung von Industrie 4.0 in KMU (Abb. 3.1).

Bislang gibt es kaum Ansätze für eine Sicherheitskultur 4.0. Insofern betritt das Vorhaben Neuland. Gleichwohl existieren eine Reihe bewährter Ansätze sowie vereinzelt Studien, die im Vorhaben genutzt bzw. aufgegriffen werden. Die Ziele des Vorhabens sind nur interdisziplinär zu erreichen durch das systematische Zusammenführen verschiedener fachlicher Perspektiven in einem gemeinsamen Bezugsrahmen. Der sicherheitskulturorientierte Transformationsansatz betrachtet das gesamte sozio-technische System von Technik, 


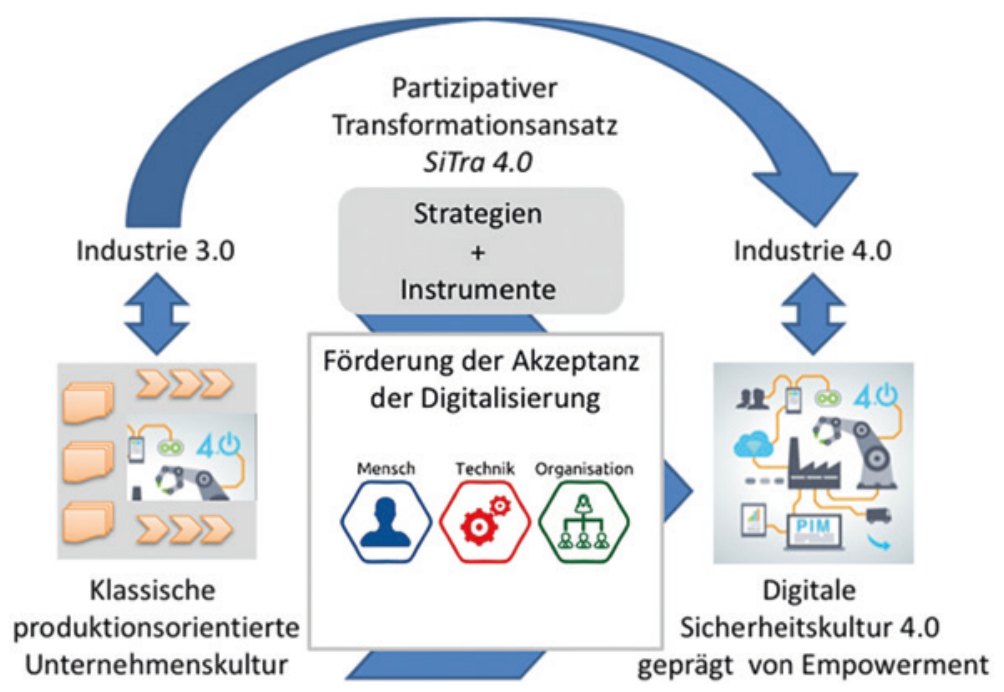

Abb. 3.1 Zielbild des Vorhabens SiTra4.0

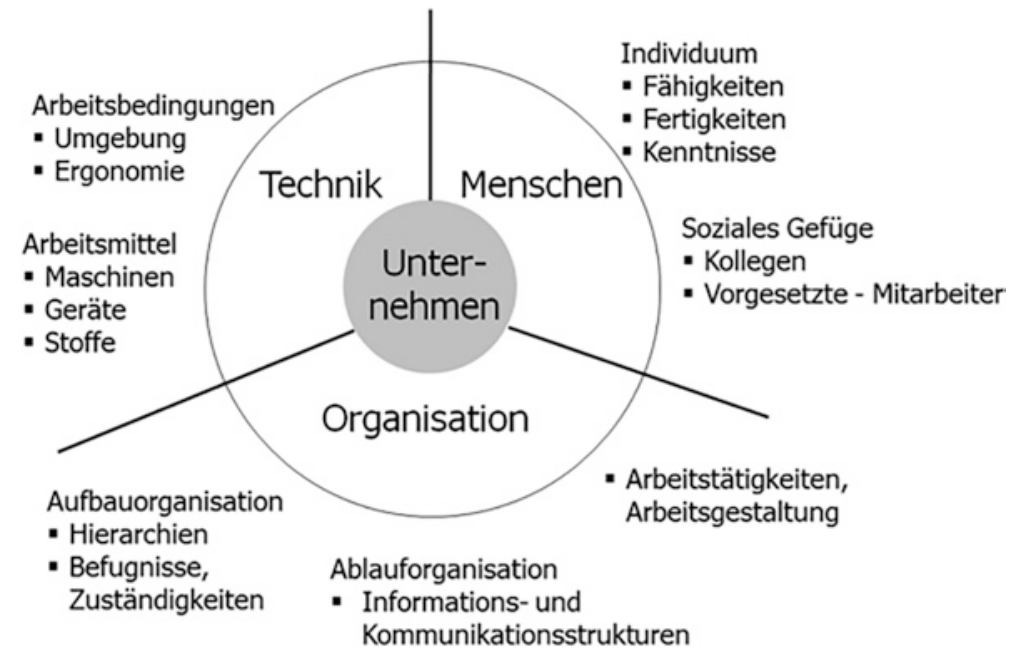

Abb. 3.2 Sozio-technisches System [3]

Organisation und Mensch (Abb. 3.2) und erlaubt einen integrativen Zugang zu vernetzten Strukturen und daran gebundene Qualifikationsanforderungen.

Der vorliegende Beitrag ist ein Ergebnis des Forschungsprojekts Nachhaltige Sicherheitskultur als Transformationsansatz für Industrie 4.0 in kleinen und mittleren Unternehmen (SiTra4.0). Das Projekt verbindet organisationsorientierte Forschung 
zu Transformationsprozessen, Studien zu Sicherheitskulturen in Organisationen und Ansätze, die sich aus sozialwissenschaftlicher Sicht mit Wandelprozesse und Sicherheitskulturen befassen. Eine wesentliche Herausforderung besteht darin, diese Perspektiven in enger Zusammenarbeit mit Praxispartnern verschiedener Branchen interdisziplinär zusammenzubringen und aufeinander zu beziehen. Wesentliche Arbeitsziele sind

- die Identifikation der Elemente bzw. Anforderungen des Arbeits- und Gesundheitsschutzes einer Sicherheitskultur 4.0, bezogen auf Empowerment, Transformation und AGS,

- die Anpassung existenter technischer Sicherheitskonzepte von KMU an die Anforderungen von Industrie 4.0 sowie

- Die Identifikation förderlicher und hemmender Faktoren beim Übergang in eine digitalisierte Arbeitswelt.

\subsubsection{Ausgangssituation und Herausforderungen in der Komponentenherstellung}

Die industrielle Arbeitswelt ändert sich schleichend, aber fundamental. Die physischen, psychischen und intellektuellen Anforderungen der modernen Arbeitswelt sind immens, die Auswirkungen der Digitalisierung und neuer Arbeitsformen schwer vorhersehbar. Unternehmens-Software wie SAP suggerieren, dass bei richtiger Anwendung und Implementierung im Unternehmen alle erfolgsentscheidenden Faktoren unter Kontrolle gehalten werden können. Der Trend zur kontinuierlichen Steigerung von sicherheitsbezogenen Features und Kontrollinstrumente führt dazu, dass 'Superspezialisten' und digitale Systeme im Fokus aller industriellen Sicherheitsbemühungen stehen. Aufgrund dieser Fokussierung erfolgte die Kontrolle von Gefährdungen bisher nur punktuell in den klassischen, analog geprägten Schutzfunktionen. Exemplarisch sind hier verschiedene Risikofelder zu nennen: Arbeitsunfall (Arbeits- und Gesundheitsschutz), Belastung und Umwelt (Umweltschutz), Belastungen durch Strahlenexposition (Strahlenschutz), Brandbelastungen (Brandschutz), kriminelle Belastungen (Objektschutz) Störungen der betrieblichen Ordnung (Werkschutz), Know-how-Verlust (Informationsschutz), störanfällige Produktion und Produkte (technischer Schutz). Insgesamt zeigt sich, dass die Beherrschung aller Risiken ein Prozess ist, der durch die Digitalisierung kontinuierlich komplexer wird.

\subsubsection{Ausgangssituation und Herausforderungen in der Baubranche}

Die Planung und Errichtung eines Gebäudes verlangt die Zusammenarbeit eines interdisziplinären Teams mit sehr unterschiedlichen Kompetenzen und Bildungshintergründen. Von planerisch-kaufmännischen Aufgaben über technische Arbeitsfelder 
hin zu handwerklichen Tätigkeiten auf der Baustelle werden sehr heterogene Arbeitsbereiche des Bauprozesses abgebildet. Neben diesen komplexen internen Unternehmensstrukturen werden die Bauvorhaben aufgrund steigender Qualitätsanforderungen sowie strengerer behördlicher Auflagen fortwährend vielschichtiger. Insbesondere für kleine und mittlere Unternehmen (KMU) wachsen dadurch die Herausforderungen für die notwendige digitale Transformation. Prominente Beispiele wie Stuttgart 21 oder der Berliner Flughafen zeigen deutlich die Auswirkungen von fehlender Übersichtlichkeit und Ineffizienzen bei Bauprozessen [22].

Seit etwa 10 Jahren kann die Baubranche lediglich ein Produktivitätswachstum von etwa drei Prozent verzeichnen. Der Zuwachs fiel in anderen Branchen deutlich höher aus. Bauspezifische digitale Ansätze, wie Building Information Modelling (BIM), finden aktuell nur zu $4 \%$ in der gesamten Baubranche Anwendung. BIM ermöglicht die digitale Abbildung aller Bauprozesse und schafft so die Schnittstelle für die Zusammenarbeit mit geringeren Reibungsverlusten der diversen Tätigkeitsfelder eines Bauunternehmens. Aufgrund der Bauprojektkomplexität sowie einer hohen Zahl an externen Beteiligten erweist sich die sukzessive Digitalisierung bislang insbesondere in KMU als sehr langwierig. Durch einen strukturierten digitalen Transformationsansatz können die bestehenden hohen Potenziale in der Baubranche erschlossen [30].

Sehr deutlich lässt sich die Herausforderung der Diversität bei der Digitalisierung der Arbeit am Beispiel der Baubranche darlegen. Aus Arbeitgebersicht stellt die Digitalisierung einen notwendigen Schritt dar, um zukunftsfähig zu sein, und gleichzeitig kann sie einen Bonusfaktor im Ringen um Fachkräfte darstellen. Dabei ist Digitalisierung nicht gleich Digitalisierung und unterliegt den Gegebenheiten der Branche und den damit verbundenen Arbeitsfeldern. Daher ist es notwendig, Digitalisierungsprozesse unter diesen - branchenspezifischen - Aspekten zu betrachten. Die Frage dabei ist, welche Aspekte die Potenziale für eine arbeitnehmerfreundliche Transformationsstrategie bieten generisch, und welche branchenspezifisch sind. Die Analyse und Bewertung dieser Aspekte die Möglichkeit, Arbeitnehmer optimal auf dem Weg in die digitale Arbeitswelt zu begleiten und gleichzeitig für Fachkräfte attraktiv zu bleiben.

\subsection{Vorgehensweise zur Zielerreichung für Unternehmen}

Im Folgenden wird die Herangehensweise für die Entwicklung und Etablierung einer Sicherheitskultur 4.0 in vier Einzelschritten dargestellt. Der erste Schritt fokussiert die Identifikation von Anforderungen an (Kultur)Transformationsprozessen in KMU und deren Implementierung auf Basis einer Ist-Analyse der zentralen Unternehmensprozesse. Im zweiten Schritt wird die bestehende Sicherheitskultur im Hinblick auf das Zusammenspiel von Arbeitssicherheit, Arbeits- und Gesundheitsschutz analysiert. Schritt drei fokussiert die Evaluation der bestehenden Sicherheitskultur, Schritt vier die Auswahl von kommunikations- und verhaltensbezogenen Maßnahmen des Empowerments von Mitarbeitern, um eine Sicherheitskultur $4.0 \mathrm{zu}$ etablieren. Schritt eins des Vorgehens 


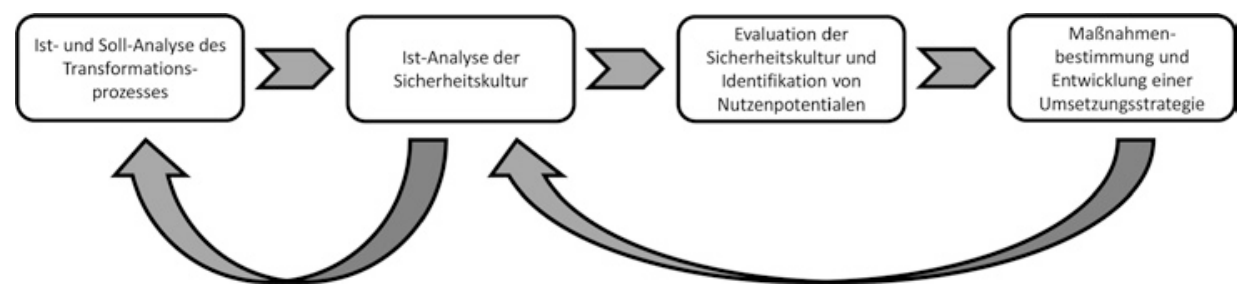

Abb. 3.3 Vorgehen bei der Entwicklung einer Sicherheitskultur 4.0

zeigt das Ziel der Transformation auf, die übrigen Schritte müssen iterativ wiederholt werden, um den Stand des Transformationsvorhabens zu monitoren, seinen Erfolg zu gewährleisten und eventuell notwendige Anpassungen an den Soll-Zustand der Transformation zu veranlassen. Das Vorgehen wird in Abb. 3.3 dargestellt und im Folgenden hinsichtlich der Einzelschritte beschrieben.

\subsubsection{Schritt 1: Anforderungen an den Transformationsprozess identifizieren}

Für die Etablierung einer Sicherheitskultur 4.0 müssen in einem ersten Schritt eine Anforderungsanalyse und eine Konzeptentwicklung für den angestrebten Transformationsprozess durchgeführt werden. Im Fokus stehen dabei die Technik und Organisation der Unternehmen. Die Umgebung wie auch die Arbeitsbedingungen im Unternehmen haben großen Einfluss auf den Erfolg einer Transformation [27]. Hier gilt es, die entscheidenden Einflussfaktoren konkret zu bestimmen und im Transformationsprozess zu berücksichtigen. Auf der organisationalen Ebene muss beschrieben werden, wie alle Beteiligten den für sich maximalen Nutzen aus der Transformation zu einer neuen Sicherheitskultur ziehen.

Für eine Anforderungsanalyse müssen die Ausprägungen „Organisationsstruktur“, „Prozesse“, Aufbau der notwendigen „Personalqualifikation“ und das zukünftige Gefüge von Rollen und „Verantwortungen“ untersucht werden, die durch „Führung“, „Kommunikation“ und „Changemanagement“ im Unternehmen verbreitet werden. Die genannten Ausprägungen sind Bestandteil des Business Modell Canvas [12]. Es ist wichtig, dass die Sicherheit im Unternehmen ständig an veränderte Rahmenbedingungen angepasst wird, um zum einen die Sicherheit im Unternehmen gewährleisten zu können und zum anderen dem Widerstand und den Ängsten der Mitarbeiter entgegenzuwirken. So kann verhindert werden, dass zu einem späteren Zeitpunkt eine radikale Umstrukturierung von Maßnahmen bezüglich der Sicherheitskultur erfolgen muss.

Der dargestellte Ansatz geht explizit davon aus, dass sich Transformationen aus strategischen Überlegungen heraus entwickeln und von diesen abgleitet wird. Demgemäß werden an einer strategischen Entscheidung und Zielsetzung ausgerichtet die weiteren Teilbereiche gestaltet. Zusätzlich werden die Gestaltungsfelder flankiert, die den derzeitigen 
Zustand der Unternehmenskultur sowie die zukünftig erwünschte Unternehmenskultur in einem definierten Zielzustand nach der Business Transformation beschreiben. Die Ergebnisse der Analysen zu Anforderungen an die Transformationsprozesse in den Praxispartnern des SiTra4.0-Projekts werden exemplarisch in Abschn. 3.3.2.1 beschrieben.

\subsubsection{Schritt 2: Die bestehende Sicherheitskultur analysieren}

In einem zweiten Schritt bei der Etablierung einer Sicherheitskultur 4.0 muss die bestehende Sicherheitskultur analysiert werden. $\mathrm{Zu}$ untersuchen sind insbesondere die einer Kultur zugrunde liegenden Grundannahmen (vgl. [25]), die als Deutungs- und Handlungsmuster maßgeblich für die tägliche Umsetzung sind. Um eine Sicherheitskultur fassbar zu machen bedarf es eines passenden Einordnungs- und Gestaltungsrahmens, der die Komplexität verschiedener Kulturaspekte über Indikatoren abbildet. Solche Kulturindikatoren müssen „Sicherheit“ und „Digitalisierung“ aufgreifen. Die Entwicklung und Anwendung eines solchen Rahmens wird exemplarisch in Abschn. 3.3.2.2 beschrieben.

\subsubsection{Schritt 3: Nutzenpotentiale einer Sicherheitskultur 4.0 offenlegen}

Der Erfolg des Transformationsansatzes muss messbar gemacht werden. Hierfür müssen geeignete Metriken identifiziert und ausgewählt werden, die den Erfolg perspektivenabhängig (Mitarbeiterrolle, Branche etc.) und zeitlich (kurz-, mittel- und längerfristige Erfolgsauswirkungen) festlegen. Dieser Schritt ist wesentlicher Bestandteil einer Nachhaltigkeitsstrategie im sicherheitskulturbezogenen Denken und Handeln im Unternehmen. Ziel muss es zunächst sein, den aktuellen Stand einer Sicherheitskultur (als Teil der Ist-Analyse) abzubilden und Nutzenpotentiale zu identifizieren. Im Laufe des Transformationsprozesses muss die Methode wiederholt angewendet werden, um Trends in der Entwicklung zu einer Sicherheitskultur 4.0 abzubilden. In SiTra4.0-Projekt wurde die Evaluation der Sicherheitskultur exemplarisch quantitativ mittels Fragebogen-Studie untersucht. Die dabei erhobenen Metriken umfassen Aspekte eines guten Arbeitsplatzes sowie motivierende und hemmende Faktoren der Digitalisierung aus Arbeitsnehmerperspektive in der Baubranche (die Ergebnisse werden in Abschn. 3.3.1.1, 3.3.1.2 und 3.3.1.3). Für die Evaluation bieten sich zusätzlich qualitative Methoden an, etwas Tiefeninterviews oder Fokusgruppen.

\subsubsection{Schritt 4: Maßnahmen für die Transformation zu einer Sicherheitskultur 4.0 ergreifen}

Der letzte Schritt einer Iteration auf dem Weg zu einer Sicherheitskultur 4.0 besteht (aufbauend auf dem Vergleich der Ist- und Soll-Analysen) in der Maßnahmenauswahl 
und der Vorgehensfestlegung. Hierzu müssen Potentiale der vorhandenen Sicherheitskultur identifiziert, Anforderungen an eine Sicherheitskultur 4.0 auf Verhaltens- und Kommunikationsebene festgelegt sowie Maßnahmen für das Empowerment von Mitarbeitern entwickelt werden.

Transformationsprozesse $\mathrm{zu}$ Industrie 4.0 erfordern Maßnahmen, die es den Beteiligten erlauben, firmeninterne Vorstellungen von Sicherheit und sicherem Verhalten $\mathrm{zu}$ verstehen und $\mathrm{zu}$ benennen, informelles und kollektives Wissen für Veränderungsprozesse produktiv zu nutzen, sich auf Stile des Umgangs und Verhaltens zu einigen sowie Strategien für die Kommunikation und gemeinsame Bewältigung von Ängsten und Unsicherheiten zu entwickeln. Dies umfasst u. a.:

- Strategien und Vorgehensweisen der Entwicklung eines unternehmensinternen Codes of Conduct (Selbstverpflichtung zu sozialem und kommunikativem Verhalten, dem Umgang mit Fehlern und kulturell sensiblen Aspekten),

- Strategien und Tools für eine intensivierte Kommunikation (die mehr ist als Informationsweitergabe) und Partizipation (z. B. Workshops, digitale Chats, Rückmeldekanäle, Teamgespräche)

- kommunikative Verfahren der industriellen Prozesserhebung [17] für die Entwicklung eines geteilten "mentalen” Gesamtbildes und eines tieferen Verständnisses für den eigenen Beitrag zum Ergebnis

- Vorgehensweisen beim eigenverantwortlichen Erkennen, Offenlegen und Bearbeiten potentieller Fehlerquellen (konstruktive Nutzung informaler Lösungsansätze, strukturierte Teamgespräche).

Veränderungsprozesse führen nicht zu den gewünschten Ergebnissen, wenn ihre Notwendigkeit nicht begründet und von den Betroffenen akzeptiert wird. Die Einführung von Maßnahmen zur Etablierung einer Sicherheitskultur 4.0 ist der kritischste Punkt im Transformationsprozess - fehlende Akzeptanz seitens der Mitarbeiter kann zum Scheitern aller Transformationsversuche führen. Die von den Mitarbeitern wahrgenommenen Risiken der industriellen Digitalisierung müssen mit geeigneten Kommunikationsformaten adressiert werden. Mit einiger Wahrscheinlichkeit ist die Bereitschaft, sich aktiv einzubringen, je nach Mitarbeiter unterschiedlich hoch. Ein vielversprechender Ansatz ist, Mitarbeiter als Promotoren für risikobewusstes Verhalten zu gewinnen. Der Auswahl- und Schulungsprozess solcher Promotoren muss von Kommunikationsexperten begleitet werden. Schulungsbedarf besteht ggf. auch auf Ebene des mittleren und höheren Managements. Eine wesentliche Aufgabe ist das Nachhalten der Umsetzung des Verhaltenskodex - etwa ob formelle und informelle Formen der kommunikativen Bearbeitung von Risiken und Fehlern konform gehen mit Sicherheitsvorschriften und -richtlinien. Darüber hinaus ist ein kontinuierliches Monitoring hinsichtlich neuer (interner und externer) Risiken erforderlich, um Richtlinien und Schulungen auf dem neuesten Stand zu halten (z. B. mithilfe von Text Mining und Topic Tracking-Methoden). 


\subsection{Ergebnisse}

\subsubsection{Auswirkungen auf Arbeitsgestaltung}

Ergebnisse zur Auswirkung der Etablierung einer Sicherheitskultur 4.0 auf die Arbeitsgestaltung umfassen Befunde zur Arbeitnehmerperspektive auf Aspekte eines guten Arbeitsplatzes (Abschn. 3.3.1.1), zu motivationalen und hemmenden Faktoren der Digitalisierung (Abschn. 3.3.1.2) sowie zu Nutzenpotentialen und Aufgabenbereichen einer sicherheitskultur-4.0-gerechten Arbeitsgestaltung (Abschn. 3.3.1.3). Diese Aspekte wurden in einer Studie der Projektpartner HCIC/Communication Science (RWTH Aachen University) und DERICHS u KONERTZ GmbH u Co KG untersucht. In der Studie wurde ein mehrstufiges Verfahren gewählt, um die Nutzenpotenziale der Arbeitnehmerperspektive zu analysieren. Zunächst wurde in einer breit angelegten Studie $(n=505)$ potenziell attraktive und hemmende Faktoren der Digitalisierung aus Arbeitnehmerperspektive erhoben. Zusätzlich wurde untersucht, welche Arbeitsfelder aus Arbeitnehmersicht im Transformationsprozess relevant sind. Neben der generischen Erhebung von Einstellungen zur Digitalisierung des Arbeitsplatzes wurde die Baubranche als Praxisbeispiel gewählt und eine weitere Studie $(n=85)$ mit Arbeitnehmern aus der Branche durchgeführt. Die Auswahl der in die Studien integrierten Aspekte erfolgte in Abstimmung mit den Projektpartnern und auf Basis von Interviews sowie Literaturstudien. Die Ergebnisse wurden trianguliert und in Zusammenarbeit mit den Praxispartnern im Hinblick auf Potenziale für eine Transformationsstrategie analysiert.

Anhand der Befunde müssen geeignete Kommunikations- und Empowermentmaßnahmen für die Transformation zu einer sicheren Arbeitsgestaltung in der Industrie 4.0 (Abschn. 3.3.1.4) entwickelt werden. Die im Teilprojekt "Entwicklung eines Befähigungs- und Beteiligungskonzeptes für eine Sicherheitskultur 4.0" vom Projektpartner HCIC/Textlinguistik und Technikkommunikation (RWTH Aachen University) mit der Westaflex $\mathrm{GmbH}$ durchgeführten Arbeiten zielten auf die Identifikation geeigneter Maßnahmen für die Etablierung einer Sicherheitskultur 4.0 in der Komponentenherstellung. Die durchgeführten Arbeiten bauen auf den Ergebnissen der Teilprojekte "Entwicklung eines Transformationsprozesses für eine Sicherheitskultur 4.0 und dessen Implementierung" (mehrdimensionales Anforderungsprofil, Heatmap

für Kernprozesse, Case-Entwicklung) und "Entwicklung eines Gestaltungsrahmens für eine Sicherheitskultur 4.0" (Kulturindikatoren) auf und wurden in Abstimmung mit den Projektpartnern durchgeführt. Die Identifizierung von Anforderungen und Barrieren erfolgte literaturbasiert sowie empirisch beim Praxispartner Westaflex sowie externen Experten. Das methodische Design nutzt einen Methodenmix (u. a. Interview, Dokumentanalyse, teilnehmende Beobachtung). Die Maßnahmenentwicklung erfolgte literaturbasiert sowie durch eine empirische Methodenkombination (u. a. Methoden der industriellen Prozessmodellierung, World Café, Tiefeninterview, teilnehmende Beobachtung, Workshops zur Einführung digitaler Dokumentationssysteme, Text Mining) und eine Re-Analyse der Ergebnisse der Anforderungsanalyse. 


\subsubsection{Ergebnisse zur Arbeitnehmerperspektive auf Aspekte eines guten Arbeitsplatzes}

Arbeitnehmerzufriedenheit stellt einen entscheidenden Faktor für gesunde Arbeitsbedingungen dar und stärkt gleichzeitig die Bindung zwischen Angestellten und Unternehmen $([23,24])$. Somit ist Arbeitnehmerzufriedenheit ein entscheidender Aspekt für eine gute Zusammenarbeit, besonders in Zeiten des Wandels.

Abb. 3.4 zeigt die Gewichtung verschiedener Aspekte eines guten Arbeitsplatzes mittels der Vergabe von Sternen $(\min =1 ; \max =10)$ durch die Probanden im Rahmen der in SiTra4.0 durchgeführten Arbeitnehmerstudie. Eine höhere Anzahl an Sternen bedeutet hier eine höhere Wichtigkeit für die Befragten. Dabei zeigt sich, dass die befragten Arbeitnehmer alle vorgelegten Aspekte als wichtig einstufen. Die meisten Sterne werden im Durchschnitt an ein gutes Arbeitsklima $(M=9,1 ; \mathrm{SD}=1,35)$, Arbeitsplatzsicherheit $(M=8,93 ; S D=1,49)$ und gesunde Arbeitsbedingungen $(M=8,93$; $\mathrm{SD}=1,57)$ vergeben. Hervor sticht hier, dass Familienfreundlichkeit im Kontrast zu den anderen Aspekten die geringste Sterneanzahl erhält $(\mathrm{M}=7,87 ; \mathrm{SD}=2,20)$.

Eine Varianzanalyse zeigte, dass die älteren Probanden (ab 56 Jahren) insgesamt signifikant mehr Sterne an die einzelnen Aspekte vergaben als die jüngeren. Die Rangfolge blieb dieselbe. Einen altersbezogenen Unterschied in Wahrnehmung und Gewichtung arbeitsplatzbezogener Facetten konnten z. B. auch Kooij et al. zeigen: Arbeitnehmer legen mit zunehmendem Alter größeren Wert auf ein angenehmes Arbeitsumfeld, während jüngere Arbeitnehmer eher Aspekte wie Aufstiegs- und Karrierepotential betonen $([20,21])$.

Arbeitnehmerfaktoren wie Alter sollten folglich als relevant in Überlegungen über die Gestaltung einer gesunden und arbeitnehmergerechten Transformation zur digitalen

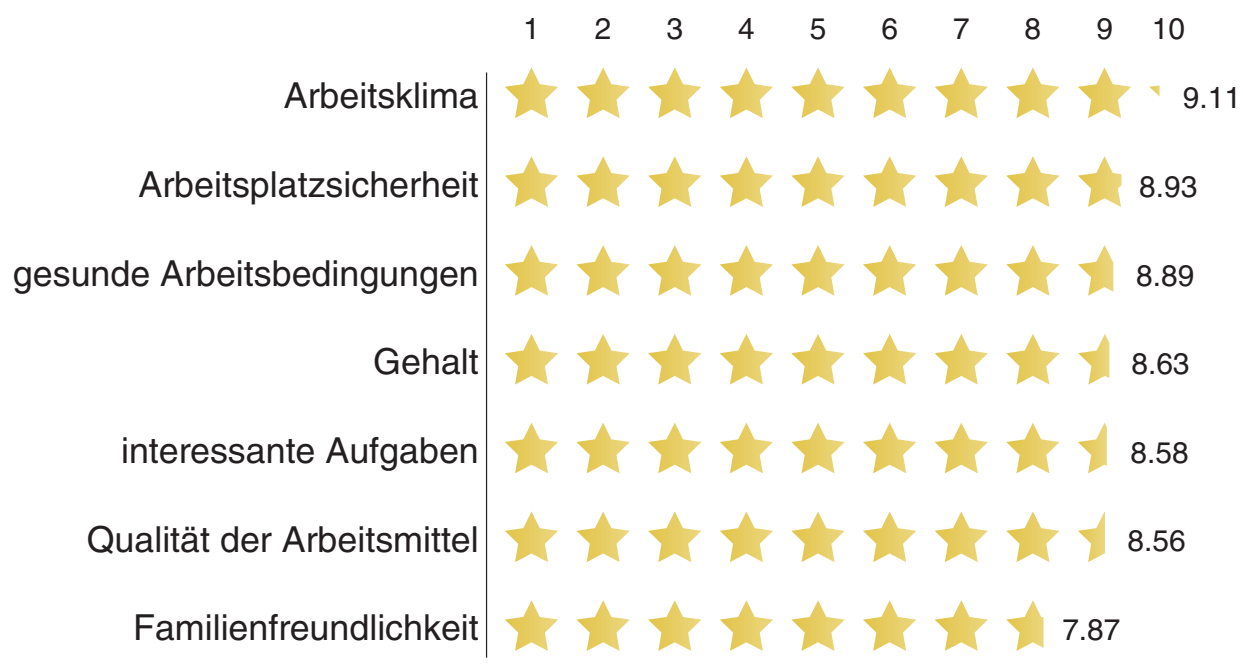

Abb. 3.4 Aspekte eines guten Arbeitsplatzes (max. mögliche Sternevergabe =10; N=505) 
Arbeit einbezogen werden. In Bezug auf die Kulturindikatoren zeigt sich hier die Relevanz der Ebene des Individuums als Unternehmensebene, die es im Rahmen einer Sicherheitskultur $4.0 \mathrm{zu}$ berücksichtigen gilt. Auch verdeutlicht das Ergebnis die hohe Relevanz von „weichen Faktoren“, wie ein gutes Arbeitsklima, für die Arbeitnehmer, die wesentlich durch die identifizierten Kulturindikatoren geprägt werden können (vgl. Abb. 3.17).

\subsubsection{Ergebnisse zu motivationalen und hemmenden Faktoren der Digitalisierung}

Grundlegende Veränderungen des eigenen Arbeitsplatzes können zu großen Verunsicherungen führen. Folgen können eine negative Haltung zum Arbeitgeber und eine Verschlechterung des Arbeitsklimas sein. Auf der anderen Seite bietet die Digitalisierung neben notwendigen Veränderungen des Arbeitgebers (Wettbewerbsfähigkeit) auch gleichzeitig viel Potenzial für positiven Wandel, wie z. B. Möglichkeiten des Empowerments, Arbeitserleichterungen und größere Autonomie der Arbeitnehmer in der Durchführung ihrer Aufgaben [1].

Für die Transformation zu einer digitalen Aufbau- und Ablaufstruktur ist es daher wichtig, Ansatzpunkte zu finden, den Mitarbeitern gezielt Sorgen zu nehmen und diesen vorzubeugen. Auf der anderen Seite müssen Potenziale und motivierende Aspekte für die Mitarbeiter in die geänderte Strategie einbezogen werden.

Die Digitalisierung hat nicht nur großen Einfluss auf die Arbeitsabläufe und -prozesse innerhalb eines Unternehmens, sondern wirkt sich gleichermaßen auch auf die Arbeitseinstellung der Mitarbeiter und die Art der Mitarbeiterführung aus. Von der Einführung einer digitalen Prozesslösung bis zur vollständigen Umsetzung und Akzeptanz durchläuft ein Unternehmen einen vier-stufigen Transformationsprozess. Abb. 3.5 bildet diese

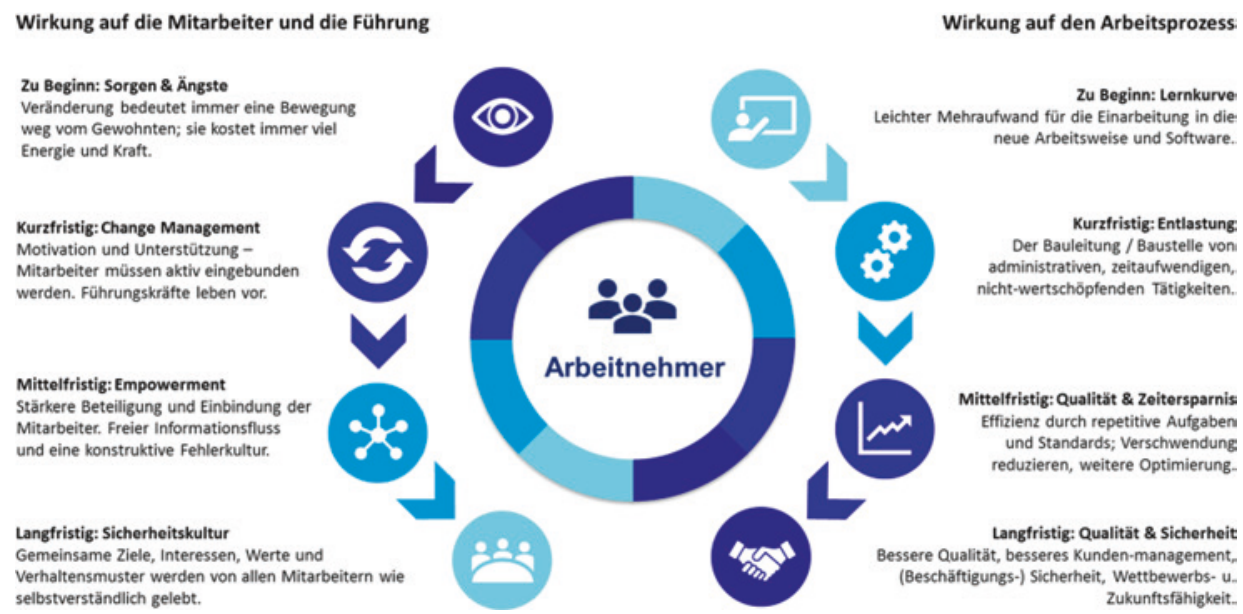

Abb. 3.5 Erwartete Auswirkung der Digitalisierung auf Arbeitnehmer in der Baubranche 
Entwicklung ab und unterscheidet zwischen der Wirkung auf die Arbeitsprozesse und der Wirkung auf die Mitarbeiter bzw. der Art der Führung.

Nach der Einführung einer digitalen Prozesslösung muss der Arbeitnehmer zunächst einen Mehraufwand betreiben, um die digitale Anwendung fehlerfrei anzuwenden und den gleichen Arbeitsoutput zu produzieren. Der Arbeitnehmer kann nun nicht mehr seiner Arbeitsroutine folgen und muss sich in neue Prozesse einarbeiten. Dies hat zur Folge, dass mehr Zeit und Energie aufgewendet werden muss, wodurch der Arbeitnehmer sich unbehaglich und unwohl fühlt.

Kurzfristig kann dann allerdings eine Entlastung von administrativen, nicht-wertschöpfenden und zeitaufwendigen Tätigkeiten festgestellt werden. In dieser Phase der ersten Entlastung müssen Arbeitnehmer aktiv in den Digitalisierungsprozess eingebunden werden und Motivation und Unterstützung der Führungskräfte erfahren. Gleichzeitig müssen Führungskräfte neue Arbeitsweisen vorleben und übernehmen in diesem Zusammenhang eine Vorbildfunktion (Change Management).

Durch die ständige Wiederholung der neu definierten digitalen Arbeitsprozesse entwickeln sich mittelfristig optimierte Anwendungsstandards und Ressourcenverschwendung wird reduziert. Arbeitnehmer werden sicherer im Umgang mit digitalen Prozessen. In dieser Phase muss ein freier Informationsfluss gegeben sein und Fehler müssen durch Führungskräfte konstruktiv toleriert werden. Nur dadurch können sich Prozesse intern weiterentwickeln und optimieren. Weiterhin sollten Arbeitnehmer verstärkt involviert und befähigt werden sich proaktiv an dem Digitalisierungsprozess zu beteiligen (Empowerment).

Nach vollständiger und umfänglicher Implementierung verbessert sich langfristig die Qualität und Sicherheit der Arbeitsprozesse. Auch Kunden profitieren dadurch und die Wettbewerbs- und Zukunftsfähigkeit des Unternehmens wird gestärkt. Zu diesem Zeitpunkt haben alle Arbeitnehmer die damit einhergehenden Verhaltensmuster, Ziele und Werte vollständig adaptiert und sehen die digitale Arbeit als selbstverständlich an.

Zum Verständnis darüber, welche Faktoren im Zuge der Digitalisierung als Potenzial und welche als eventuelle Belastung durch die Arbeitnehmenden wahrgenommen werden, wurde daher die Wahrnehmung von Einzelaspekten vertiefend untersucht. Die Ergebnisse der Befragung von Arbeitnehmern zeigte, dass Potenziale vor allem in der Verbesserung der eigenen Arbeitsleistung qualitativ $(M=3,95 ; S D=1,47)$ - und quantitativ $(\mathrm{M}=3,93 ; \mathrm{SD}=1,44)$ gesehen werden.

Aber auch eine verbesserte Kommunikation innerhalb des Unternehmens wird als potenzielle Verbesserung durch die Digitalisierung wahrgenommen $(M=3,8$; $\mathrm{SD}=1,46)$. Vorteile durch zeitliche Flexibilisierung der Arbeit $(\mathrm{M}=3,11 ; \mathrm{SD}=1,62)$ oder eine verbesserte Work-Life-Balance $(\mathrm{M}=3,08 ; \mathrm{SD}=1,55)$ finden ebenfalls Zustimmung bei den Befragten (vgl. Abb. 3.6).

Die hier von den Arbeitnehmern als besonders positiv wahrgenommenen Aspekte bieten sich an, um diese durch Akzentuierung im Rahmen eines Veränderungsprozesses zu implementieren und dadurch das Engagement der Arbeitnehmer zu erhöhen. Bezogen auf die Kulturindikatoren spiegeln sich diese Aspekte vor allem auf der Gruppenebene 


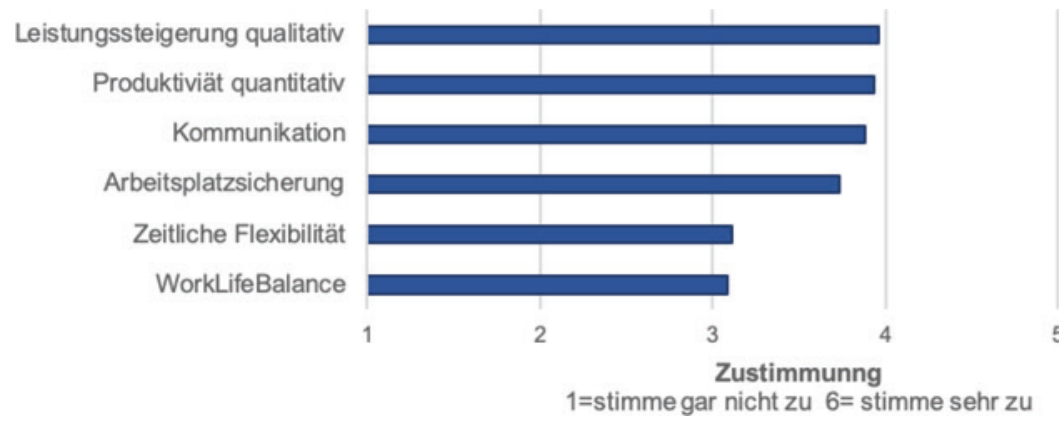

Abb. 3.6 Arbeitnehmerwahrnehmung der Potenziale der Digitalisierung $(\mathrm{N}=505)$

des Kulturindikators Kommunikation (v. a. Schnittstellen) wider, aber auch Werte spielen hier eine wesentliche Rolle im Sinne von Leitlinien (z. B. gute Work-Life-Balance als Wert im Unternehmen, vgl. Abb. 3.17).

Die durchgeführte Befragung in der Baubranche ergab ebenfalls eine insgesamt positive Haltung zu den unterschiedlichen Digitalisierungspotentialen (vgl. Abb. 3.7). Auch hier erhalten Motive, die die eigene Leistung und eine Verbesserung der Arbeitsperformanz betreffen, die höchste Zustimmung (neue Potenziale: $\mathrm{M}=4,87$; $\mathrm{SD}=0,81$; Produktivität: $\mathrm{M}=4,83$; $\mathrm{SD}=0,97)$. Hier wird darüber hinaus der Aspekt der Vernetzung zwischen den relativ separierten Arbeitsbereichen, die in der Baubranche vorherrschend sind, akzentuiert $(M=4,71 ; S D=0,78)$. Aufgrund dieser sehr diversen Arbeitsbereiche ist die Analyse der abteilungsabhängigen Bewertung der Potenziale durch die Digitalisierung von Interesse für eine passgenaue Transformationsstrategie, um der diversen Abteilungsstruktur im Unternehmen gerecht zu werden.

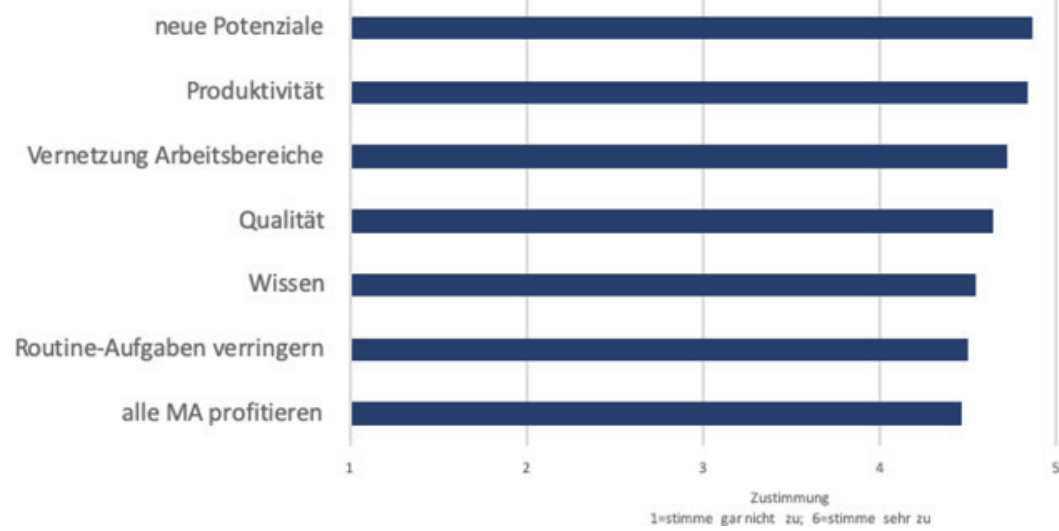

Abb. 3.7 Arbeitnehmerwahrnehmung der Potenziale der Digitalisierung am Beispiel Baubranche $(\mathrm{N}=85)$ 


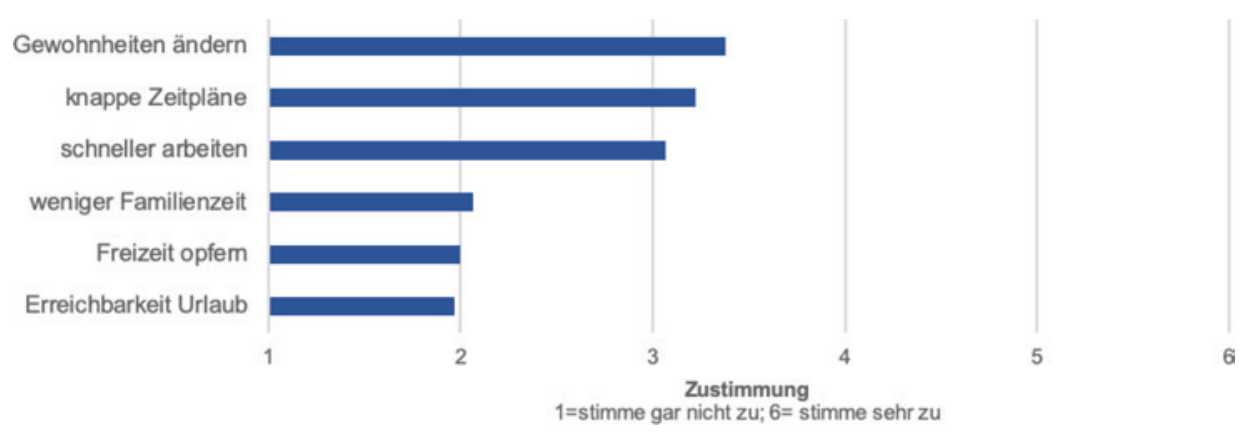

Abb. 3.8 Arbeitnehmerwahrnehmung der Potenziale der Digitalisierung nach Abteilungen $(\mathrm{N}=85)$

Die großen Unterschiede zwischen den Bereichen Handwerk, technisch sowie planend/kaufmännisch/ administrativ, spiegeln sich auch in der Wahrnehmung der motivationalen Aspekte wider (vgl. Abb. 3.8). Während sich für den technischen Bereich vor allem neue Potenziale durch die Digitalisierung als Motivator zeigen $(M=5,06$; $\mathrm{SD}=0,76$ ), bewerten die im Bereich Planung, Administration und kaufmännische Abteilung tätigen Teilnehmer die Steigerung der eigenen Arbeitsqualität durch die Digitalisierung am positivsten $(\mathrm{M}=4,00 ; \mathrm{SD}=1,00)$. Für die handwerklich tätigen Arbeitnehmer stellt der Aspekt der besseren Wissensdistribution im Unternehmen das größte Potenzial dar $(\mathrm{M}=4,45 ; \mathrm{SD}=0,69)$.

Sowohl die generische als auch die spezifische Erhebung zeigen, dass vor allem die Verbesserung der eigenen Leistung sowie die Produktivität als positiv wahrgenommen und somit als motivierender Treiber in der Transformation zur digitalen Arbeit betont werden. Auch Vernetzung und Kommunikation werden als besonders positiv wahrgenommen. Es zeigt sich aber auch, dass die motivierenden Aspekte nach Arbeitsbereich unterschiedlich bewertet werden und somit, dass der Einbezug der Diversität der Abteilungen in die Ausgestaltung von Maßnahmen und Strategien Potential für eine erfolgreiche Transformation bietet.

Zusätzlich zu motivierenden Aspekten der Digitalisierung aus Arbeitnehmersicht wurden auch potenziell hemmende Faktoren untersucht.

Auf allgemeiner Ebene zeigt sich, dass die generelle Wahrnehmung negativer Aspekte eher gering ausfällt (vgl. Abb. 3.9). Zustimmung findet hier vor allem die Sorge vor Änderungen der eigenen Arbeitsgewohnheiten durch die Digitalisierung und den damit verbundenen geänderten Arbeitsabläufen $(M=3,39 ; \mathrm{SD}=1,50)$. Auch Befürchtungen, dass mit den Änderungen knappere Zeitpläne $(M=3,22 ; S D=1,48)$ und ein schnelleres Arbeitspensum $(M=3,07 ; \mathrm{SD}=1,54)$ einhergehen, werden in der Tendenz bestätigt. Sorgen, dass durch die Digitalisierung das Privatleben leidet, etwa durch weniger Zeit mit der Familie $(M=2,06 ; S D=1,47)$, Einschnitte in die Freizeit $(\mathrm{M}=2,0 ; \mathrm{SD}=1,30)$ oder die Erwartung an Erreichbarkeit für den Beruf im 


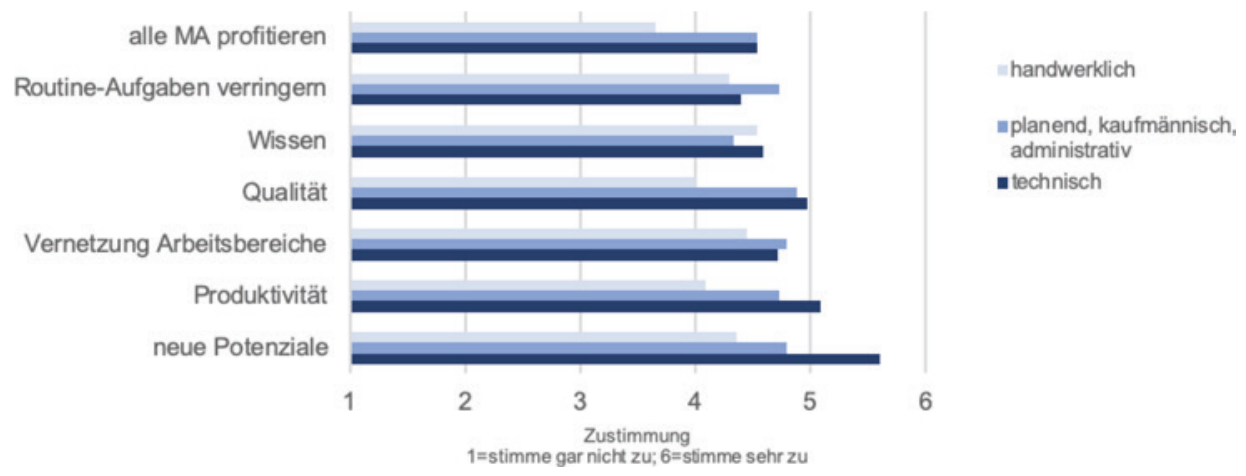

Abb. 3.9 Arbeitnehmerwahrnehmung negativer Aspekte Digitalisierung $(\mathrm{N}=505)$

Urlaub $(\mathrm{M}=1,98 ; \mathrm{SD}=1,48)$ werden eher schwach wahrgenommen. Somit zeigt sich auch hier, wie bei den motivierenden Faktoren, eine klare Fokussierung auf die Auswirkungen der Digitalisierung auf die eigene Arbeitsperformanz. Die Ergebnisse weisen auf das Potenzial des Kulturindikators Einbindung hin, im Sinne von Trainings, die in geänderten Arbeitsabläufen schulen, um hier Belastungen vorzubeugen (vgl. Abb. 3.17).

Die Erhebung in der Baubranche zeigt, dass hier Sorge besteht, dass durch die Einführung digitaler Arbeitsprozesse die eigene Leistung stärker kontrolliert werden könnte $(\mathrm{M}=3,81 ; \mathrm{SD}=1,08)$. Auch die Befürchtungen vor erhöhtem Arbeitsaufwand durch geänderte Arbeitsabläufe und neu zu erlernenden Systemen finden hier Ausdruck (3,43; $\mathrm{SD}=1,15)$.

Zusätzlich zeigt sich am Beispiel Baubranche, dass die Befragten eher zustimmen, die soziale Interaktion unter den Mitarbeitern könnte abnehmen $(M=3,34 ; S D=1,05)$. Hingegen wurde hier im Kontrast zur generischen Befragung eine geringe Sorge vor mehr Fehlern in der eigenen Arbeitsperformanz $(M=2,92 ; S D=0,91)$, Arbeitsplatzverlust $(\mathrm{M}=2,74 ; \mathrm{SD}=1,16)$ oder einer negativen Änderung der Arbeitsgewohnheiten $(\mathrm{M}=2,11 ; \mathrm{SD}=0,83)$ deutlich (vgl. Abb. 3.10).

Der Vergleich der potenziell negativen Aspekte der Digitalisierung des eigenen Arbeitsplatzes nach Abteilungen zeigte für das Beispiel Baubranche, dass die Wahrnehmung sich deutlich nach Abteilung unterscheidet (vgl. Abb. 3.11). Wie bei den motivationalen Aspekten wird deutlich, dass die handwerklich tätigen Arbeitnehmer in ihrer Einschätzung deutlich von den anderen Arbeitsbereichen abweichen. Für die handwerklich Tätigen ist die Sorge vor Leistungskontrolle $(\mathrm{M}=4,45 ; \mathrm{SD}=1,03)$, mehr Arbeitsaufwand $(\mathrm{M}=4,16 ; \mathrm{SD}=0,75)$, aber auch die Abnahme sozialer Interaktion $(\mathrm{M}=4,00 ; \mathrm{SD}=0,89)$ deutlich stärker ausgeprägt als bei Arbeitnehmern der anderen Bereiche. Auffällig ist hier auch die stärker ausgeprägte Sorge vor dem Verlust des Arbeitsplatzes $(\mathrm{M}=3,81 ; \mathrm{SD}=1,07)$.

Die Unterschiede in der Bewertung der Aspekte liegen in der Art der Tätigkeit und den stark diversen Arbeitsbereichen der Baubranche. Dennoch zeigt sich am Beispiel der 


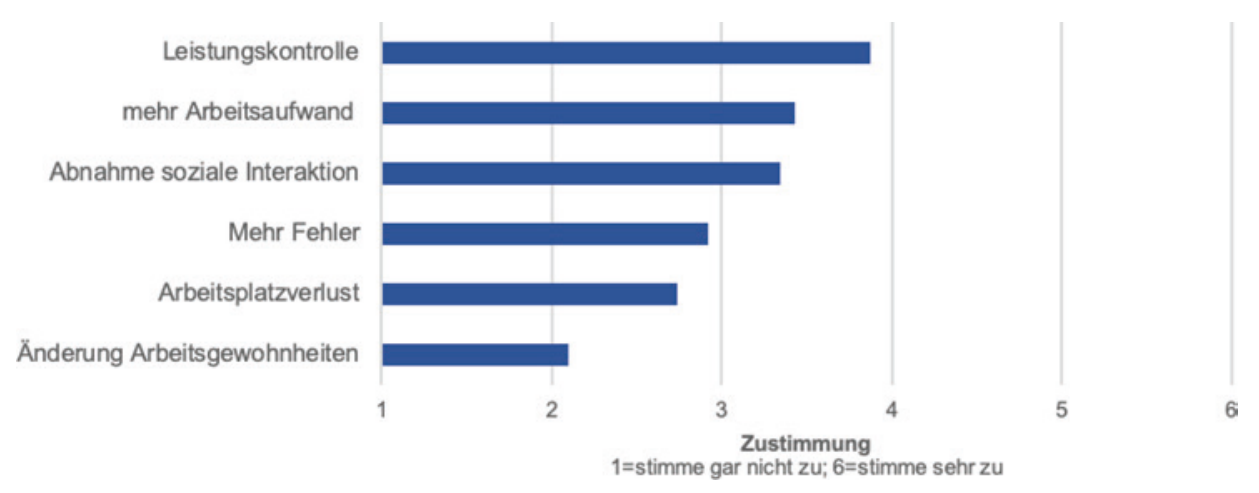

Abb.3.10 Arbeitnehmerwahrnehmung negativer Aspekte Digitalisierung, Beispiel Baubranche $(\mathrm{N}=85)$
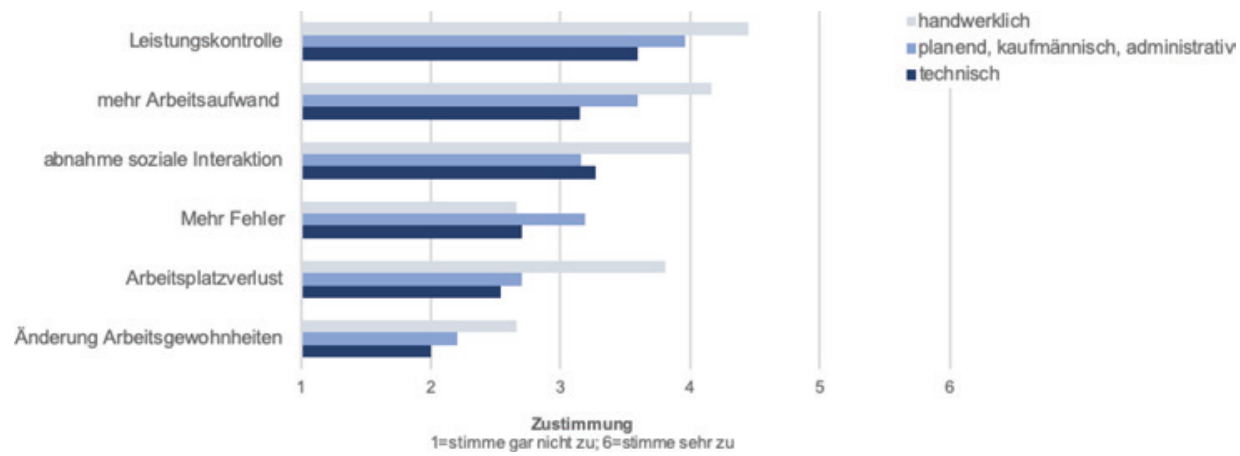

Abb. 3.11 Arbeitnehmerwahrnehmung negativer Aspekte Digitalisierung nach Abteilungen $(\mathrm{N}=85)$

Baubranche deutlich die Wichtigkeit des Einbezugs der diversen Arbeitsbereiche und die Notwendigkeit des Verständnisses über unterschiedliche Motivations- und Befürchtungsstrukturen. Somit wird hier die Bedeutsamkeit der Gruppenebene des Rahmenmodells Sicherheitskultur 4.0 deutlich (vgl. Abb. 3.17). Erfolgreiche und für den Mitarbeiter gesunde Transformationsstrategien müssen dies berücksichtigen, um ein gesundes Arbeitsklima zu schaffen und Mitarbeiter so im Wandel zu einer digitalen Unternehmensstrategie einzubeziehen und die sich dadurch bietenden Potenziale für Arbeitnehmer und Unternehmen bestmöglich auszuschöpfen.

Während die generische Studie zeigen konnte, dass grundlegende Wahrnehmungen von hemmenden und motivierenden Faktoren vor allem aufgaben- und leistungsbezogene Aspekte betreffen, zeigte die spezifische Studie in der Baubranche, dass es feine, aber unter Umständen gewichtige Unterschiede in der Bewertung und Wahrnehmung potenziell belastender und motivierender Faktoren innerhalb eines Unternehmens gibt. Weitere Ergebnisse im Rahmen des Projekt SiTra4.0 verdeutlichten, dass auch andere Faktoren, wie z. B. das Alter hierbei eine wichtige Rolle spielen 
können (vgl. [16, 18]). Somit weisen die vorliegenden empirischen Ergebnisse auf die im Rahmenmodell Sicherheitskultur 4.0 identifizierten Kulturindikatoren hin und verdeutlichen die Relevanz der darin einbezogenen Unternehmensebenen (vgl. Abb. 3.17).

\subsubsection{Ergebnisse zu Nutzenpotentialen und Aufgabenbereichen einer sicherheitskultur-4.0-gerechten Arbeitsgestaltung}

Auf der Basis der Ergebnisse der Umfragen, Literaturstudien, Interviews und Workshops wurden im Rahmen des Projekts SiTra4.0 vier wesentliche Aufgabenbereiche identifiziert, innerhalb derer Stellschrauben für eine arbeitnehmergerechte Transformation zur Digitalisierung vorhanden sind.

Abb. 3.12 zeigt vier Aufgabenbereiche, die in SiTra4.0 als relevant identifiziert wurden. Diese beziehen sich auf strategische Unternehmensbereiche, die auf den Menschen als

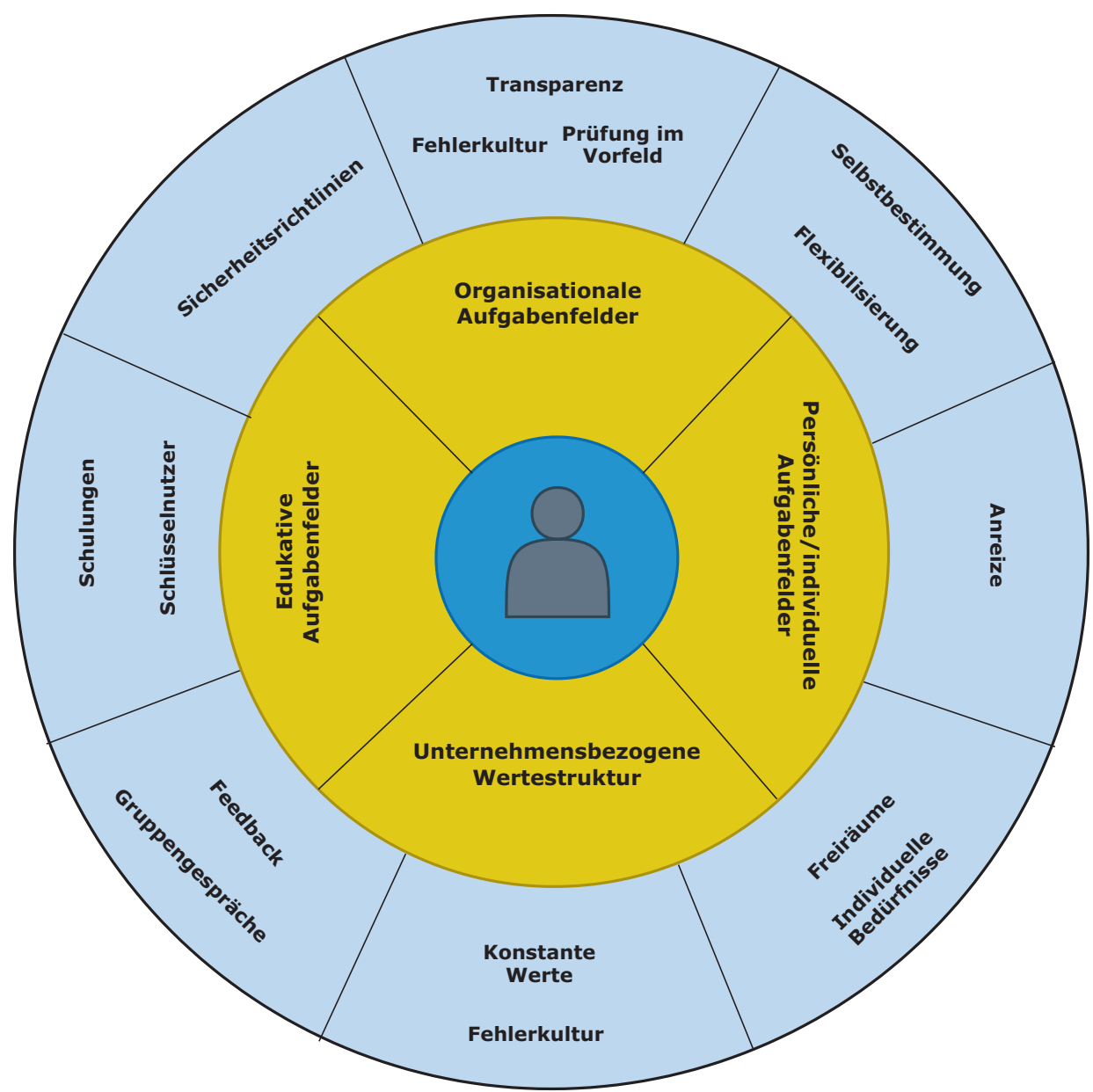

Abb. 3.12 Nutzenpotentiale und Aufgabenbereiche der digitalen Transformation am Arbeitsplatz 
Arbeitnehmer in seinem Arbeits- und Aufgabenumfeld einwirken. Von diesen Aufgabenbereichen ausgehend, zeigt der äußere Ring Handlungsbereiche, aus denen heraus konkrete Maßnahmen für eine Transformationsstrategie abgeleitet werden können. Aus dem edukativen Aufgabenbereich leitet sich so z. B. das Handlungsfeld Schulungen ab. Aus allen durchgeführten Umfragen geht hervor, dass die Arbeitnehmenden sich Schulungen wünschen, die entweder die neu eingeführten Systeme oder die geänderten Arbeitsabläufe umfassen. Darauf basierend können konkrete Schulungsmaßnahmen entwickelt werden, die die empirischen Ergebnisse und Analysen einbeziehen.

Die Aufgabenbereiche und Handlungsfelder sind nicht trennungsscharf und überlappen sich. Sie dienen als Orientierungsrahmen für die weitere Entwicklung und Evaluation von Transformationsmaßnahmen auf der Basis der empirischen Ergebnisse. Weitere Studien werden adressieren, ob und inwiefern die Aufgabenbereiche und Handlungsfelder die Transformation in verschiedenen Branchen beeinflussen und welche Rolle dabei Branchenspezifika und Arbeitnehmerdiversität spielen.

In SiTra4.0 wurden die als generisch identifizierten Aufgabenbereiche und Handlungsfelder mit den Praxispartner validiert. Aus Sicht der Baubranche liefern alle vier Aufgabenbereiche einen maßgeblichen Beitrag für die erfolgreiche Umsetzung der digitalen Transformation. Jedoch ist in der Ausgestaltung der Maßnahmen darauf zu achten, dass die Komplexität aufgrund der hohen Arbeitnehmerdiversität und der Vielzahl externer Beteiligter im Bauprozess sehr schnell zunimmt. Daher fokussiert SiTra4.0 den Kernwertschöpfungsprozess der Bauleitung. Klassischerweise laufen sämtliche Schnittstellen für die termin-, qualitäts- und kostengerechte Bauleistung unter Berücksichtigung der Sicherheitsanforderungen in der Bauleitung zusammen.

Bezogen auf die identifizierten Handlungsfelder kommt der Schaffung von persönlichen Anreizen eine besondere Rolle zu. Im Vordergrund der Digitalisierung bestehen die maßgeblichen Anreize der Bauleitung in einer Entlastung und Glättung des Stresslevels im täglichen Arbeitsprozess auf der Baustelle. Als Grundlage für eine erfolgreiche Transformation muss die unternehmensbezogene Wertestruktur zudem klar definiert sein. Beim Prozess der Bauausführung bedeutet dies beispielsweise, dass es eindeutige Standards geben sollte, an denen sich die Bauleitung orientieren kann. Gibt es Abweichungen von diesen Standards, gilt es, diese genau zu analysieren und Anpassungen vorzunehmen. Hier profitiert der Wandel von einer gelebten Fehlerkultur, in der Abweichungen offen kommuniziert werden und somit alle Mitarbeiter daraus lernen können und das Unternehmen sich langfristig verbessern kann. Durch Standards werden der Bauleitung Entscheidungen abgenommen - es entsteht mehr Freiraum für wichtige, individuelle Aufgabenfelder und somit eine höhere Prozess- und Arbeitssicherheit.

Im Bereich der Organisationalen Aufgabenfelder ist es empfehlenswert, die Mitarbeiter in den Transformationsprozess mit einzubeziehen. Erfolgen eine frühzeitige Prüfung bzw. Testung neuer Prozesse und Technologien durch die Mitarbeiter, die diese später umsetzen, kann eine bessere Qualität des neuen Prozesses und eine höhere Akzeptanz durch die Mitarbeiter erzielt werden. Dies führt wiederum zu einer erhöhten Erfolgswahrscheinlichkeit der Transformation. 
Neue Technologien und Arbeitsinhalte müssen den Mitarbeitern durch Lernangebote zugänglich gemacht werden. Dies erfolgt optimalerweise in Form von Schulungen (persönliche Ebene) sowie der Ausbildung sogenannter Schlüsselnutzer, die die neuen Fähigkeiten in die Breite tragen können.

In allen Bereichen ist eine offene Kommunikation sowie Feedback erfolgskritisch. Nur so kann das Unternehmen einen Wandel nicht nur auf dem Papier, sondern in den Köpfen und gelebten Verhaltensweisen - als Kultur - vollziehen.

\subsubsection{Ergebnisse zur Wirksamkeit von Kommunikations- und Empowermentmaßnahmen für die Transformation zu einer sicheren Arbeitsgestaltung in der Industrie 4.0}

Der vorliegende Beitrag fasst wichtige Befunde des Projektpartners HCIC/Textlinguistik und Technikkommunikation ([3-5]) zusammen, die beim Praxispartner Westaflex erhoben wurden. Zunächst wurde das Potential der vorhandenen Sicherheitskultur in Bezug auf Kommunikation und Empowerment untersucht. Anschließend wurden Anforderungen an Kommunikation und Empowerment für die Etablierung einer Sicherheitskultur 4.0 identifiziert und passende Maßnahmen exemplarisch angewendet.

Westaflex konzentriert sich in seiner Digitalisierungsstrategie auf den Einsatz neuer Produktions- und Logistiktechnologien, womit sich Strukturverschiebungen der Qualifikationsanforderungen ergeben und sich die Arbeitsinhalte verändern. In diesem Kontext ist die Gewährleistung der Sicherheit (d. h. die gezielte Kontrolle der genannten Risikofelder) als ganzheitliches System im Unternehmen wenig verbreitet. Lediglich bei erkennbar hohen Sicherheitsrisiken und Behördenanforderungen kommen Beauftragte, im Einzelfall Bevollmächtigte, zum Einsatz. Weitere Herausforderungen betreffen die Aus- und Weiterbildung der Mitarbeiter: Der durch neue Digitaltechnologien erzielbare Flexibilitätszuwachs tritt aufgrund unzureichender Qualifikation der Mitarbeitenden nicht ein. Gleichzeitig ist die Integration von Zeit- und Migranten-Arbeitsverhältnissen in die in die sich wandelnden Arbeitsanforderungen und -kontexte nur mit hohen Mehraufwänden zu leisten. Auf technischer Seite entstehen infolge der Implementierung integrierter Assistenzsysteme zum Teil Systeminkompatibilitäten zwischen Unternehmensorganisation und technischen Systemen. Um die genannten Herausforderungen zu bewältigen, fördert Westaflex die Entwicklung eines ganzheitlich integrierten, vernetzten Schutzsystems auf Basis einer Sicherheitskultur für Industrie 4.0, die Lohngerechtigkeit sowie Motivations- und Leistungsanreize bietet.

Die durchgeführten Analysen deuten auf ein geteiltes, unternehmensweites Verständnis von Sicherheit hin. Ein Großteil der Mitarbeiter nennt in den Interviews eine hohe gefühlte Sicherheit, die sich aus formellen Maßnahmen (z. B. zur Reduktion von Gefahren) wie auch informellen Maßnahmen (z. B. dem konstruktiven Ton bei der Kommunikation von Fehlern und Risiken zwischen Mitarbeitern) ergibt. Die Dokumentenanalyse zeigt, dass umfangreiche Regelwerke die Grundlage für sicheres Arbeiten im Betrieb legen. Regeln für sicherheitsgerechtes Verhalten umfassen Anweisungen zur Weitergabe von sicherheitsrelevanten Informationen, zur Absprache 
zwischen Mitarbeitern, zu eigenverantwortlichem Handeln auf Mitarbeiterseite, zur Dokumentation von Fehlern und zum Absolvieren sicherheitsrelevanter Trainings. Die Analyse zeigt jedoch auch, dass Anweisungen häufig vage formuliert sind und selten auf Rollen oder Aufgaben eingehen, Medien für die Informationsweitergabe und Kommunikation (z. B. Melden von Schäden) nur in Ausnahmefällen explizit benennen und Schrittfolgen der Aneignung bzw. Verbreitung von Wissen [3] außer Acht lassen. Die Befunde lassen sich zum Teil auf die Rechtsverbindlichkeit von Anweisungen (und damit verbundene potentielle Teilhaftbarkeiten) zurückführen (alles, was schriftlich fixiert ist, kann im Streitfall genutzt werden). Ein zweiter Grund betrifft Kompetenzen und Fähigkeiten beim Formulieren von Anweisungen. Die Verfasser sind in der Regel eher technische Experten; gerade in schriftlichen Kontexten zeigt sich Weiterbildungsbedarf. Das betriebliche Regelwerk wird den Mitarbeitern in Unterweisungen durch Kollegen und jährlichen Sicherheitsschulungen durch externe Experten veranschaulicht. Die Interviewstudien zeigen, dass die Mitarbeiter diese Maßnahmen schätzen, insbesondere weil das Regelwerk als zu umfangreich wahrgenommen wird, das Wissen um betriebliche Regeln der regelmäßigen Auffrischung bedarf und Schulungen Rückfragen, etwa zur Aktualität von Anweisungen und technologischen Änderungen ermöglichen. Insgesamt zeigt sich ein geteiltes Verständnis arbeitsbezogener Risiken (z. B. Quetschungen und Schnitte bei der Arbeit an Maschinen). In den Beschreibungen arbeitsbezogener Risiken zeigt sich sehr deutlich ein implizites Verständnis des formalen Regelwerkes und seiner Sinnhaftigkeit.

Ein bisher eher wenig genutztes Potential und ein deutlicher Handlungsbedarf zeigen sich insbesondere auf der Ebene abteilungsinterner Sub-Sicherheitskulturen. Arbeitsbezogene Risiken werden von Mitarbeitern abteilungsbezogen unterschiedlich eingeschätzt und behandelt, z. B. Verstöße gegen Schutzkleidungsanordnungen (etwa das Tragen bzw. Nichttragen von Handschuhen bei Tätigkeiten, die Fingerspitzengefühl erfordern). Im engeren Arbeitsumfeld achten die Kollegen verstärkt auf Sicherheit und diskutieren Gefahrenquellen mit anderen; dazu gehören auch Kollegen, die sich normalerweise nicht in diesem Arbeitsumfeld aufhalten. Gleichzeitig wird Abteilungsdenken in der Weitergabe sicherheitsrelevanter Informationen bemängelt: Während die Weitergabe sicherheitsbezogener Informationen abteilungsintern gut funktioniert, fehlt der abteilungsübergreifende Austausch zu Problemen, die früh in der Produktionskette auftreten und die nachfolgende Arbeit anderer behindern wie auch zu später in der Prozesskette entstehenden Problemen, die Mehrarbeit erzeugen, z. B. durch das Nachbessern von Produkteigenschaften. Die Entwicklung passgenauer Maßnahmen für (Kultur)Transformationsprozesse ist nur möglich auf Basis einer sorgfältigen und konsequenten vergleichenden Analyse abteilungsinterner Subkulturen und übergeordneter betriebsinterner Sicherheitskultur.

Die Ergebnisse der Analysen werden genutzt, um auf bestehenden positiven Eigenschaften der aktuellen Sicherheitskultur aufzubauen und in den Schritten 1 bis 3 identifizierten Anforderungen an die Entwicklung einer Sicherheitskultur 4.0 zu erfüllen. 
Die Fallstudie identifiziert eine Reihe von Anforderungen bei der Entwicklung von Sicherheitskultur für Industrie 4.0, die bei der Wahl von Kommunikationsmaßnahmen berücksichtigt werden müssen. Ein wesentliches Problem ist die Divergenz der Schnelligkeit des technologischen Wandels und der Langsamkeit kultureller Veränderungsprozesse (sich ändernde Überzeugungen und Einstellungen). Gerade aber letztere spielen eine entscheidende Rolle und müssen deshalb in Transformationsprozessen besonders sorgfältig geplant und unterstützt werden. Die Studie zeigt, dass Konzepte, die sich rein auf Arbeits- und Gesundheitsschutzaspekte beschränken, in diesem Kontext zu kurz greifen. AGS ist Teil eines komplexen sozio-technischen Gefüges, in dem das gemeinsame Handeln immer wieder überprüft und diskursiv hinterfragt werden muss. Dies gilt für Bereiche wie wahrgenommene Sicherheit, Produktsicherheit, Datenschutz, Maschinensicherheit oder Prozesssicherheit. Eine Sicherheitskultur 4.0 setzt ein Sicherheitsverständnis voraus, das alle Aspekte der täglichen Arbeit (z. B. rechtliche Konsequenzen, ergonomische Aspekte, Kommunikation von Fehler- und Gefahrenquellen) umfasst und neue Risiken infolge der Digitalisierung proaktiv bearbeitet. Die Grenze zwischen technologisch Machbarem und sozial-ethisch Vertretbarem muss immer nachhaltig diskursiv ausgehandelt werden. Die Befragten nennen als Beispiel den Einsatz von Sensoren, die es erlauben, Maschinenausfälle vorherzusagen, anderseits aber auch die Überwachung der Arbeitseffizienz von Mitarbeitern ermöglichen.

Die Digitalisierung führt $\mathrm{zu}$ erheblichen Veränderungen im Arbeitsleben, wie z. B. orts- und zeitungebundene Arbeitskontexte und daran gebundene Praktiken und Schnittstellen. Risikobewusstes Handeln erfordert von den Mitarbeitern fundierte Kenntnisse potenzieller Risiken in digitalisierten Produktionsprozessen wie auch die Möglichkeit und den Willen, sich in Entscheidungsfindungsprozesse einzubringen, um maßgeschneiderte Sicherheitsstrategien zu entwickeln. Empowerment ist eine wesentliche Voraussetzung für eine Sicherheitskultur, die auf verschiedenen Ebenen des Unternehmens greift: lokal, auf mittlerer Managementebene bis zur globalen Unternehmensebene.

Die Implementierung einer Sicherheitskultur 4.0 setzt eine sorgfältige Planung von Sicherheitsstrategien voraus, bevor Transformationsansätze in die Praxis umgesetzt werden. Die Planung muss partizipativ erfolgen, d. h. unter Einbeziehung des Erfahrungswissens und der Lösungsansätze derer, die die Arbeit leisten. Insbesondere bei intelligenten Maschinen müssen vor der Inbetriebnahme entsprechende Maßnahmen durch alle Betroffenen (z. B. Maschinenführer, Vorgesetzte, Management) entwickelt und ratifiziert werden. Die Mitarbeiter müssen umfassend über Ziele, Vorteile und vor allem Risiken der geplanten Transformation informiert werden sowie Bedenken und Widerstände berücksichtigt werden.

Eine wesentliche Herausforderung und Bedingung für die Etablierung einer Sicherheitskultur 4.0 ist die für alle nachvollziehbare Formulierung von Werten und darauf bezogenen Verhaltensanforderungen in einem Verhaltenskodex für Mitarbeiter. Solche 
Regelwerke müssen im Verlauf des Transformationsprozesses immer wieder überprüft und den sich verändernden Bedingungen angepasst werden.

(Schriftlich verdauerte) Festlegungen erfüllen nur dann ihren Sinn, wenn sie nicht als statisches „Bestandswissen“ vorgehalten werden, sondern Teil horizontaler Informationsflüsse und Besprechungen (entlang von Produktionsketten) sind. Dies setzt das Überdenken traditioneller Kommunikationskanäle voraus. In dem betrachteten Unternehmen fordern die Befragten beispielsweise eine "papierlose Produktion": Laufkarten sollen z. B. nicht länger als Ausdrucke zirkulieren, sondern elektronisch auf Tablets abrufbar sein. Die Umstellung würde ermöglichen, risikobezogene Handlungsanweisungen rollen- und kontextbezogen aufzubereiten und „on demand“ zur Verfügung zu stellen, das Nachschlagen in Regelwerken entfällt.

In allen Zusammenhängen zeigt sich, dass dem Management und Führungskräften eine zentrale Rolle in der Kommunikation und beim gemeinsamen Erarbeiten von Lösungen zukommt. Dies gilt dezidiert für kontroverse Themen wie auch die Einführung und Gestaltung einer (neuen) Sicherheitskultur 4.0. Es reicht nicht, Veränderungen in den Unternehmenszielen zu benennen, aus Sicht der Mitarbeiter interessiert vor allem, welche Anforderungen und Konsequenzen sich daraus für sie ergeben - etwa im Sinne unternehmensintern gewünschter sicherheitsrelevanter Verhaltensweisen wie auch unternehmensexternen Handelns (z. B. formelles und informelles Darstellen des Unternehmens und seiner Werte nach außen an Dritte). Dies gilt insbesondere für den Risikobereich: Führungskräfte müssen mit gutem Vorbild vorausgehen, indem sie sich selbst konsequent an Sicherheitsregeln halten und sicherheitsbezogenes Handeln von anderen aktiv einfordern. Letzteres umfasst die Forderung darauf zu achten, dass Partnerunternehmen Sicherheitsstandards einhalten und nicht in Drittländern zu menschenunwürdigen Bedingungen produzieren lassen.

Eine zweite wesentliche Bedingung für den Erfolg von Transformationsprozessen ist das Einbeziehen der Mitarbeiter und ihr Empowerment. Die Befragten thematisieren wiederholt, dass Sicherheitsverbesserungen auch bottom-up geschaffen werden müssen. Die Mitarbeiter sollten zu Innovatoren werden, die sich aktiv in Transformationsprozesse einbringen und Lösungen für aufkommende Sicherheitsprobleme entwickeln und bottom-up initiieren. Sie sollen Änderungsprozesse aktiv fordern und beschleunigen. Die Befragten thematisieren insbesondere monotone, belastende Aufgaben und alternative Formen ihrer Bearbeitung (z. B. Ausführung durch Roboter). Im Zusammenhang damit sehen sie Bedarf im Überdenken von Rollen und Aufgabenprofilen sowie den Bedarf nach Qualifizierungsmaßnahmen. Das Management soll mitarbeitergetriebene Initiativen ernst nehmen, fördern und lenken. Dies erfordert Wertschätzung, Aufmerksamkeit und organisationales Zuhören. Mitarbeiterbeteiligung darf nicht nach einem "Black-Box-Prinzip" erfolgen - Mitarbeiter erwarten Feedback zum Stand der Bearbeitung ihres Vorschlags, zum Entscheidungsfindungsprozess (etwa Angaben zu Gründen für die Annahme oder Ablehnung) und zur Umsetzung des Vorschlags. Sie wollen über Vorschläge ihrer Kollegen informiert werden und diese ggf. weiterbearbeiten 
- insgesamt zeigt sich ein hohes Interesse an (der Kommunikation von) Co-CreationProzessen.

Für die Etablierung einer Sicherheitskultur 4.0 wurde ein mehrstufiges Verfahren entwickelt und beim Praxispartner Westaflex erprobt. Das Vorgehen zielt darauf, in einem ersten Schritt mit adäquaten Maßnahmen die geplante Einführung digitaler Systeme an die Mitarbeiter zu kommunizieren und Hinweise zu deren Einfluss auf Sicherheitsaspekte zu erhalten. In einem zweiten Schritt werden Mitarbeiter dazu befähigt, an der Entwicklung der geplanten Systeme partizipieren zu können, um deren Akzeptanz und Praxistauglichkeit zu gewährleisten. Im Folgenden werden zwei Beispiele zur Umsetzung der gewählten Methoden gegeben und konkrete Auswirkungen auf das Unternehmen beschrieben.

- Kommunikation: Mittels der Managing-by-walking-around-Methode wurden Mitarbeiter an ihren Arbeitsplätzen über die Einführung eines neuen Dokumentationssystems gezielt bezogen auf ihre Rollen, Aufgaben und Arbeitskontexte informiert. Das System soll auf Tablets mobil und flexibel im Arbeitsalltag eingesetzt werden können und die papierbasierten Laufkarten im Unternehmen digitalisieren. Die Methode erbrachte umfangreiche Hinweise zu Potentialen wie Grenzen der Einsatzmöglichkeiten des Systems. Gleichzeitig zeigte die Methode sicherheitsbezogene Probleme und Lösungsansätze auf.

- Empowerment: Um schon vor der Einführung des digitalen Dokumentationssystem dessen Praxistauglichkeit zu erproben, wurden Mitarbeiter aktiv in die Entwicklung des Systems eingebunden und Teilhabe an darauf bezogenen Entscheidungsfindungsprozessen ermöglicht. So wurden beispielsweise Maschinenführer verschiedener Abteilungen gebeten, das System auf einem Tablet im laufenden Betrieb zu erproben und Rückmeldungen zu Optimierungspotentialen zu geben. Die Methode des Co-Developments durch tatsächliche Nutzer erbrachte Hinweise auf benötigte Funktionen und Inhalte sowie abteilungsspezifische Anforderungen an das System.

Die Maßnahmen zeigen konkrete Auswirkungen auf die Kultur sowie Arbeitsgestaltung des Unternehmens: Mitarbeiter berichten von einer Aufbruchsstimmung gegenüber digitalen Lösungen - wahrgenommene Verbesserungen betreffen u. a. die Gestaltung von Arbeitsplätzen und Arbeitsbedingungen sowie Möglichkeiten der Höherqualifizierung (beispielsweise vom Maschinenführer zum Prozessmanager).

\subsubsection{Produktivitätswirkung bzw. Wirtschaftlichkeitsbetrachtung}

Die Praxispartner konnten durch die Implementierung des Sicherheitskultur4.0-Konzeptes ihre „digitale Reife“ steigern und die Wettbewerbsfähigkeit ausbauen. Es zeigt sich, dass ein geteiltes Sicherheitsverständnis sowie erhöhte Aufmerksamkeit 
gegenüber Sicherheitsbelangen zwischen den Mitarbeitern Probleme wie Risiken verringern und die Produktivität steigern. Maßgebend für positive Produktivitätswirkungen der Etablierung einer Sicherheitskultur ist zum einen die Gestaltung der Transformationsprozesse zu einer Industrie 4.0 (Abschn. 3.3.2.1), die vom Forschungsinstituts für Rationalisierung (FIR) an der RWTH Aachen bei den Praxispartnern Westaflex und DERICHS u. KONERTZ untersucht wurden. Zum anderen ist die Analyse und Entwicklung der etablierten Sicherheitskultur notwendig (Abschn. 3.3.2.2). Dieser Teilbereich wird maßgeblich vom Projektpartner CBM GmbH bearbeitet.

\subsubsection{Ergebnisse zu notwendigen Transformationsprozessen einer Industrie 4.0}

Um Anforderungen an Transformationsprozesse bei den Praxispartnern zu ermitteln, wurde zunächst ein Anforderungskatalog mit den im Business Transformation Canvas [12] identifizierten Gestaltungsfeldern erarbeitet. Eingangs wurde seitens des FIR in Zusammenarbeit mit den beteiligten Praxispartnern die jeweilige Ist-Situation bezüglich einer Sicherheitskultur 4.0, also die Verzahnung von Digitalisierung und Arbeitssicherheit, aufgenommen und analysiert. Hierfür wurden jeweils zwei Workshops bei dem Praxispartner Westaflex durchgeführt. Anhand des Business Model Canvas (vgl. Abb. 3.13) wurden die Geschäftsprozesse erhoben und das Geschäftsmodell der Unternehmen skizziert und für das Projekt definiert. Wie eingangs erwähnt, ist es für einen Transformationsansatz, unabhängig von der Ausprägung eines Unternehmens, unumgänglich, das vorherrschende Geschäfts- bzw. Wertschöpfungsmodell zu verstehen und als Ausgangspunkt der Transformation zu sehen.

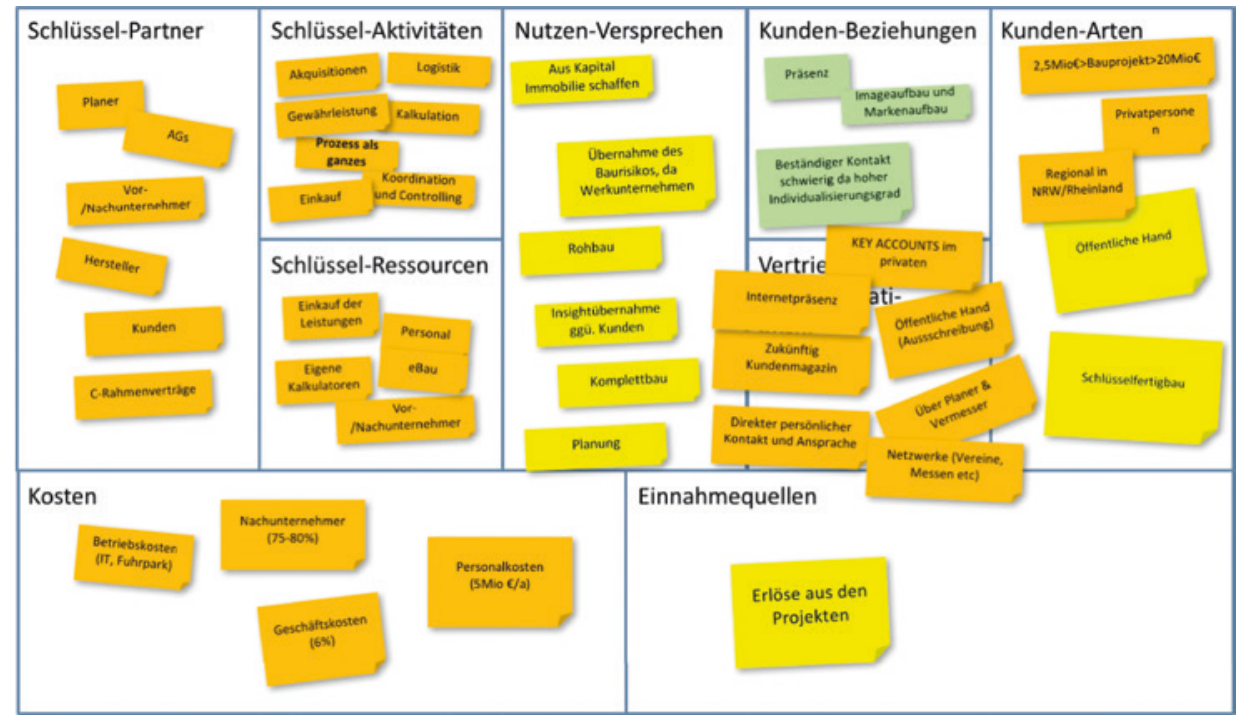

Abb. 3.13 Business Modell Canvas der Firma Westaflex 
Anhand von Experten und Gruppeninterviews wurde die vorherrschende Sicherheitskultur sowie der Stand der Digitalisierung in den betrachteten Prozessen erfasst. Die Ergebnisse wurden so aufgearbeitet, dass die Integration in ein mehrdimensionales Anforderungsprofil erfolgen kann (vgl. Auszug in Tab. 3.1). Dazu wurden die Anforderungen des Transformationsprozesses mit denen der Sicherheitskultur 4.0 und der Verhaltens- und Kommunikationsebene verglichen. Der Anforderungskatalog stellt

Tab. 3.1 Auszug Anforderungskatalog Führung

\section{Kommunikation auf einer persönlichen Beziehungsebene}

Das stärkste Mittel, Menschen von einem wichtigen Anliegen zu überzeugen bzw. zu einer Veränderung zu bewegen, ist das direkte Gespräch. Dementsprechend sollten Mitarbeitergespräche und kleine GruppenMeetings in den Arbeitsalltag integriert werden. So können u. a. Verbesserungsvorschläge gesammelt und in regelmäßigen Abständen angepasst werden

\section{Kenntnisse über Sensoren und Aktoren}

Führungskräfte, die Prozesse digitalisieren wollen, sollten wissen, wie Sensoren und Aktoren im Allgemeinen funktionieren. Dies erweitert die Entscheidungsoption hinsichtlich der benötigten Daten für die Digitalisierung der abzubildenden Prozesse., wie Sensoren und Aktoren im Allgemeinen funktionieren

\section{Kooperative Führung}

Technologischer Wandel und zunehmend individuelle Kundenanforderungen zeigen insbesondere in Industrieunternehmen die Grenzen der Einbeziehung von Mitarbeiterinnen und Mitarbeitern in formalisierten bürokratischen Prozessen auf. Ein entscheidendes Erfolgskriterium von Führung wird daher die Art der Kommunikation im Unternehmen mit angepassten Kommunikationstechnologien und -formen sein, da sie eine erfolgreiche Differenzierung vom Wettbewerb ermöglichen kann
Hohe Lern- und Veränderungsbereitschaf

Die hohe Entwicklungsdynamik digitaler

Technologien erfordert von Führungskräften und Beschäftigten eine erhöhte Lern- und Veränderungsbereitschaft. Dies erfordert nicht nur einen Wandel im Denken des Einzelnen, sondern auch in der gesamten Unternehmenskultur. Eine aktivierende betriebliche Präventionskultur entscheidet wesentlich über die Qualität der Gestaltung der digitalen Transformation

Entwicklung der Leistungsfähigkeit von Mitarbeiterinnen und Mitarbeitern

Der zunehmende Einsatz cyber-physischer Systeme erfordert eine Zunahme der technischen Anforderungen, auch bei Personalentwicklung und Rekrutierung. Die Entwicklung der Beschäftigten im Unternehmen, Selbstständigkeit und Weiterqualifizierung verändern sich ebenfalls. Für Führungskräfte bedeutet dies, dass die Motivierung und Entwicklung der Leistungsfähigkeit von Mitarbeitern und Mitarbeiterbindung eine wichtige Rolle spielt. Gleiches gilt für die Führungskräfte und die Entwicklung ihrer Führungskompetenzen

\section{Gesundheitsförderliche Führung}

Die Krankheitsquote kann verringert werden, wenn mindestens $50 \%$ der Mitarbeiter eine App für Rückenbildung benutzen und diese mehrmals am Tag für ungefähr 5 min nutzen. Die Befähigung, die eigene Gesundheit und die Gesundheit der Mitarbeiter angemessen zu regulieren, sichert neben dem Erhalt der Arbeits- und Leistungsfähigkeit, auch den Unternehmenserfolg 
den Ordnungsrahmen für die Transformation hin zu einer Sicherheitskultur dar. Anhand dessen soll nicht nur die Organisation einen Wandel erfahren, sondern auch die Unternehmenskultur transformiert und die Mitarbeiterkompetenzen gestärkt werden. Es wurde dabei unterschieden zwischen:

- Anforderungen an die Führung (Kommunikation auf einer persönlichen Beziehungsebene, hohe Lern- und Veränderungsbereitschaft, Kenntnisse über Sensoren und Aktoren, Entwicklung der Leistungsfähigkeit von Mitarbeiterinnen und Mitarbeitern, kooperative Führung, gesundheitsförderliche Führung),

- Anforderungen an den Mitarbeiter (Fachkompetenz, Methodenkompetenz, Sozialkompetenz, personale Kompetenz, professionaler Umgang mit Ängsten, Vermittlung der langfristigen Ziele, Potenziale eines Mitarbeiters erkennen und fördern, Konfliktmanagement, Stressmanagement, Zielharmonisierung, Vermeidung divergenter Rollen) und

- Anforderungen an die Organisation (Unternehmenstransformation, Unterstützung durch Personalbeschaffung, Verbesserung der kognitiven und qualitativen Anforderungen, Umgang mit Zeit- und Leistungsdruck, gesundheitsförderliche Unternehmenskultur, Unterstützung durch ein Vergütungssystem, Unterstützung durch ein Personalcontrolling, Abteilungsunterschiede, Erfahrungsunterschiede, Verständlichkeit, gemeinsamer Austausch, Umsetzbarkeit, Branchenübertragbarkeit).

Der industrielle Paradigmenwechsel der Industrie 4.0 veranlasst eine intensivere Weiterbildung und Kompetenzstärkung der Mitarbeiter eines Unternehmens. Der Umgang mit der neuen digitalisierten Arbeitswelt erfordert nicht nur eine zukunftsorientierte Kompetenzentwicklung, sondern ist auch die Basis für eine gesellschaftliche Teilhabe im Zeitalter der Digitalisierung. Personale Kompetenz beinhaltet die Entwicklung von produktiven Einstellungen, Werthaltungen, Motiven und Selbstbildern.

Um den Handlungsrahmen für SiTra4.0 zu komplementieren, wurde in mehreren Erhebungen eine Heatmap für Kernprozesse der Praxispartner Westaflex und DERICHS u KONERTZ erstellt. Sie dient als Rahmen für das weitere Vorgehen im Projekt und ermöglichte, in den Unternehmen ausgewählte Prozesse genauer zu betrachten und „Cases“ für SiTra4.0 zu identifizieren. Anschließend wurden für die Unternehmen die identifizierten Prozesse und Anforderungen an den Transformationsprozess zusammengefasst. Die Unternehmen wurden unter zwei Aspekten betrachtet. Zum einen wurde bewertet, wie sich die Arbeitsumgebung bzw. der Arbeitsablauf durch die Digitalisierung verändert, zum anderen wurde die Auswirkung auf den Arbeits- und Gesundheitsschutz in einzelnen Prozessschritten bewertet. Anhand des graphischen Tools konnten handlungsrelevante Felder bzw. Prozesse in den Unternehmen identifiziert werden. Dafür wurde für die Praxispartner der aus dem Business Modell Canvas abgeleitete Wertschöpfungsprozess abgebildet. Zur Bewertung wurden die bekannten Dimensionen der MTO-Analyse [29] herangezogen. Die erste Dimension Mensch-Maschine bildet den 
direkten Kontakt zwischen Menschen und Maschinen ab. Sie wird häufig als Benutzerschnittstelle bzw. User Interface bezeichnet. Die zweite mit dem Heatmaptool erhobene Dimension ist die Schnittstelle zwischen Technik und Organisation. Sie betrachtet die Interaktion des Unternehmens (organisierte Menge von Menschen) mit Technologien, um ein gewünschtes Ergebnis zu erzielen. Die dritte Dimension erfasst die Schnittstelle Technik und Organisation, also das Zusammenspiel in einem soziotechnischen System. Zur Bewertung der Prozessschritte wurde zunächst mit den Unternehmen der Digitalisierungsgrad in den einzelnen Dimensionen anhand einer Heatmap analysiert (Abb. 3.14). Dabei bedeutet die grüne Füllung einen niedrigen, die gelbe Füllung einen mittleren und die rote Füllung einen hohen Grad an Digitalisierung. Beispielhaft kann hier der digitalisierte Auftragseingang über eine Eingabemaske im Gegensatz zur händischen Aufnahme am Telefon aufgeführt werden. Anschließend wurden alle Felder hinsichtlich der sich neu ergebenen Arbeitssicherheit innerhalb der Prozessschritte analysiert und bewertet. Eine entscheidende Frage ist: „Verändert sich meine Arbeitsumgebung so sehr, dass sich auch der Einfluss auf meine Gesundheit ändert?“. Ein gutes Beispiel sind veränderte gesundheitliche Bedingungen durch ständige Erreichbarkeit, etwa wenn der Arbeitnehmer in seiner Freizeit einen Blick auf dienstlichen E-Mails wirft oder ein berufliches Telefonat führt.

Abb. 3.14 zeigt beispielhaft eine Heatmap für den Prozess Bauleitung. Die hier dargestellte Heatmap orientiert sich an den 7 Phasen der HOAI (Honorar Ordnung für Architekten und Ingenieure), die ein Projektentwickler für seine Kunden im Kernprozess „Objekterstellung“ bedient - Grundlagenermittlung, Vorplanung, Entwurfsplanung, Genehmigungsplanung, Ausführungsplanung, Vorbereitung der Vergabe, Mitwirkung bei der Vergabe, Objektüberwachung sowie Objektbetreuung und Dokumentation - und wandelt diese für das Projekt ab. Da der Prozess mit den vielen beteiligten Subunternehmen (Gewerke) und Fachkräften im Unternehmen schwer erfassbar ist, fokussiert das Forschungsvorhaben den Teilprozess „Bauleitung“. Die Bauleitung (nach HOAI: „Objektüberwachung“) leitet die Baustelle und ist für den ordnungsgemäßen Ablauf der Objekterstellung verantwortlich. Durch seine dezentrale Arbeit ist der Bauleiter prädestiniert für das Forschungsprojekt, da er sich stark zwischen der analogen (Baustelle) und der scheinbar digitalen (Büro) Welt bewegt und alle Schnittstellen bedienen kann und muss.

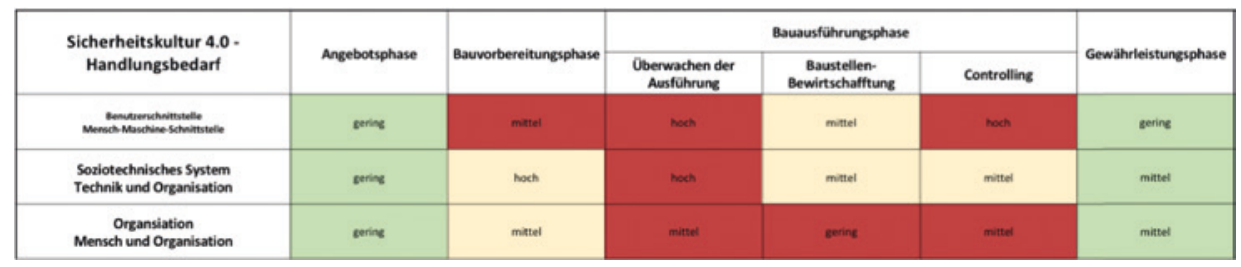

Abb. 3.14 Veränderte Heatmap von DERICHS u KONERTZ 
Den Abschluss der Anforderungsanalyse und Case-Entwicklung bildete die Integration der Ergebnisse in ein mehrdimensionales Anforderungsprofil. Anhand der identifizierten Prozesse der Wertschöpfungskette sowie der aufgenommenen Anforderungen wurde ein Transformationsprozess für eine Sicherheitskultur 4.0 und deren Implementierung erarbeitet.

Für die Entwicklung der Umsetzungsstrategie wurden die Fähigkeiten der Praxispartner zur Umsetzung der Transformation bewertet, um ein individuelles Vorgehen zu ermöglichen und dieses anschließend zu verallgemeinern. Dafür wurden die Prozesse hinsichtlich ihres Digitalisierungsgrades analysiert. Anhand von Interviews und Workshops konnten so die Prozessschritte mit den größten Potentialen wie auch Hemmnissen für eine Kultur in der Digitalisierung identifiziert werden. Die Ergebnisse wurden in einer Prozesslandkarte (vgl. Abb. 3.15) festgehalten und visualisiert. Die Wertschöpfungsprozesse der Praxispartner wurden zunächst hinsichtlich der vier Digitalisierungsbausteine „Business Intelligence“, „Cloud-Computing“, „Mobile Endgeräte“ und der „Schnittstellen“-Thematik bewertet. Die Bausteine dienen als Referenz, wie digital ein bestimmter Prozessschritt im betrachtenden Unternehmen ist und ob in den Ausprägungen Potential für den Nutzen bestimmter Bausteine vorhanden ist. So kann bewertet werden, welche Trendthemen der Digitalisierung im Unternehmen noch nicht oder nur in geringem Maße umgesetzt wurden. Darauf aufbauend wurden die wertschöpfenden Tätigkeiten auf der prozessualen Ebene bewertet. Anhand von Interviews kann so der aktuelle Ist-Zustand im Workflow beurteilt werden.

Durch die Gewichtung der Ergebnisse wurden entscheidende Handlungsfelder aufgedeckt, um anschließend geeignete Maßnahmen für die Umsetzung ableiten zu können. Anhand der identifizierten Prozesse der Wertschöpfungskette der beiden Praxispartner wurde gezielt ein Transformationsprozess für eine Sicherheitskultur 4.0 und dessen Implementierung erarbeitet. Einzelne, signifikante Prozessschritte wurden

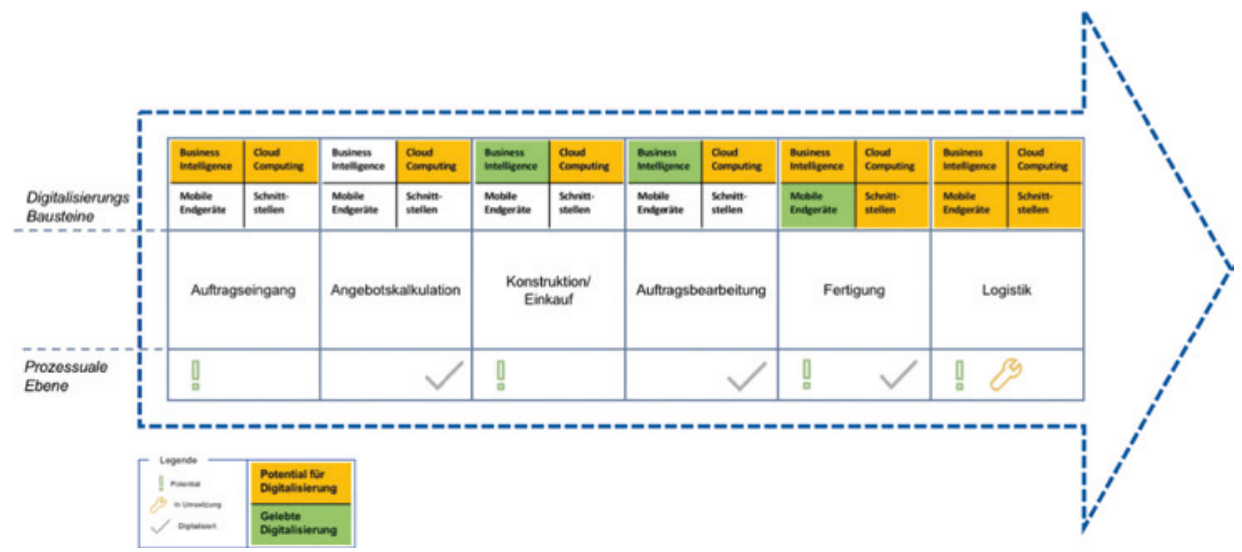

Abb. 3.15 Digitalisierungsgrad der Prozessschritte von Westaflex 
genauer analysiert, Soll- und Ist-Zustände definiert und der integrative Strategie- und Maßnahmenkatalog punktuell umgesetzt. Die abgeleiteten Transformationspfade wurden verallgemeinert und die Erkenntnisse zu Anforderungen an eine Sicherheitskultur 4.0 für das Design und die Konzeption des Transformationsprozesses.

\subsubsection{Ergebnisse zur Transformation der Sicherheitskultur}

Im Teilvorhaben "Entwicklung eines Gestaltungsrahmens für eine Sicherheitskultur 4.0" wurde ein Rahmenmodell basierend auf Kulturindikatoren [2] entwickelt. Anhand theoretischer Annahmen (u. a. [9-11, 13-15, 25]) und Daten von Unternehmen wurden fünf übergeordnete relevante Kulturindikatoren einer Sicherheitskultur 4.0 definiert: Werte, Führung, Kommunikation, Einbindung und Regelung. In die Betrachtung werden alle Unternehmensebenen einbezogen: (1) die organisatorische Ebene, auf der Ziele, Strategie, Richtlinien und Regeln festgelegt werden, (2) die Gruppen-Ebene, auf der Regeln, Ziele und Richtlinien ausgearbeitet und in betrieblichen Handlungsanweisungen und Vorgehensweisen überführt werden und (3) die individuelle Ebene, auf der Ziele, Regeln und Richtlinien im Arbeitsalltag angewandt und in der ,betrieblichen Realität“ konkretisiert werden. Abb. 3.16 erfasst Manifestationen der genannten Kulturindiatoren für verschiedene Unternehmensebenen. Der Indikator Werte manifestiert sich auf der Ebene der Organisation z. B. in den Unternehmenszielen, etwa in Leitlinien wie „Sicherheit hat immer Vorrang“. Auf der Ebene Gruppe manifestieren sich Werte in teilweise dokumentierten Normen, wie „Wir fangen erst mit der Arbeit an, wenn es sicher ist“", auf der individuellen Ebene in Einstellungsformulierungen wie „Ich passe auf mich und meine Kollegen auf“. Die Anwendung des Rahmenmodells wird in Abschn. 3.3.2.2 anhand von Projektergebnissen veranschaulicht.

Um Anforderungen an eine Sicherheitskultur 4.0 im praktischen Umfeld ermitteln zu können, wurde seitens CBM in den Unternehmen der Praxispartner der Status der

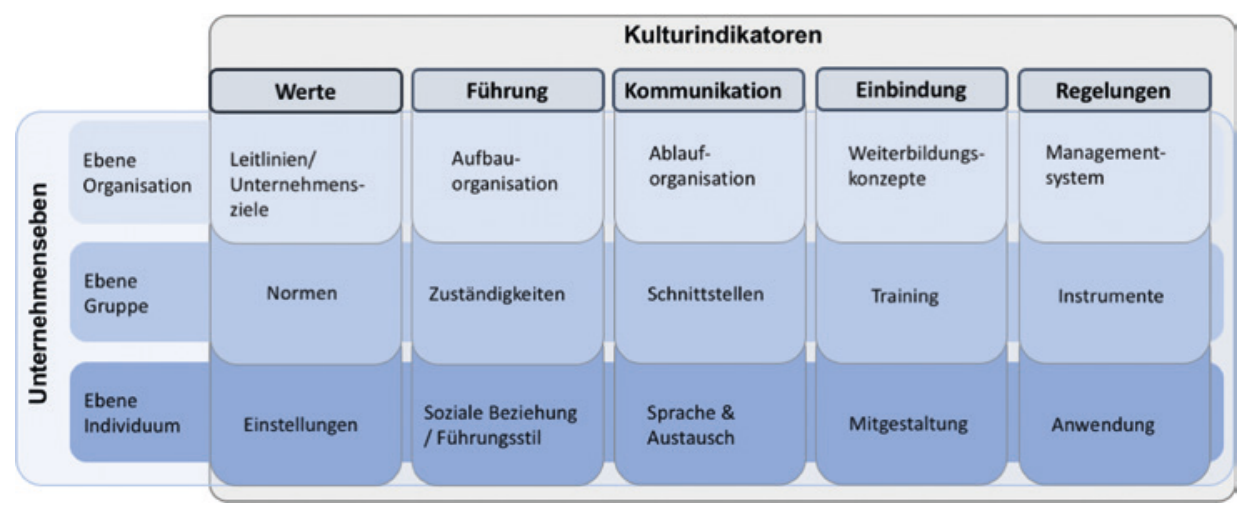

Abb. 3.16 Rahmenmodell Sicherheitskultur 4.0 - Manifestation der Kulturindikatoren auf Unternehmensebenen 
Umsetzung von AGS und Sicherheitskultur erhoben. Die Systematik der Erfassung folgte inhaltlich dem Rahmenmodell der Kulturindikatoren. Da der Kern der Kultur die Grundannahmen - nicht direkt erfassbar ist, fokussierte die Erhebung die Ebene der geäußerten Werte und der Artefakte und schloss von diesen auf Grundannahmen. Die „gewünschte“ Sicherheitskultur des Unternehmens lässt sich anhand der Dokumente und formalen Kommunikation des Unternehmens (Zielbilder, Leitlinien, Regeln) sowie von Aussagen der Führungskräfte und Mitarbeiter erfassen; die „tatsächliche“ Kultur ist anhand der Analyse von Arbeitshandlungen und Zuständen im Unternehmen rekonstruierbar.

Methodisch wurde die gewünschte formale Sicherheitskultur anhand von Dokumentenrecherchen und ergänzenden Experteninterviews mit verantwortlichen Führungskräften und Sicherheitsfunktionen er-fasst. Betrachtet wurde die Dokumentation der Aufbauorganisation (Organigramm, Management-handbuch, Pflichtenübertragung), der Ablauforganisation (Managementhandbuch, Qualifizierungs-/Weiterbildungspläne, Prozessbeschreibungen, Organisation) und der AGS Dokumentation (Unfallstatistiken, Gefährdungsbeurteilungen, Betriebsanweisungen, Unfalluntersuchungen, Meldung Beinahe-Unfälle, Auditberichte, Unterweisungsunterlagen, Begehungsprotokolle). Die Erhebung der Artefakte erfolgte durch Begehungen vor Ort (Ziel: Erfassung der Umsetzung des AGS in den Unternehmen). Betrachtet wurden Zustände, die Anwendung von Sicherheitsinstrumenten sowie die Umsetzung von Regeln und Verhaltensweisen im betrieblichen Alltag, die die Sicherheitskultur wiederspiegeln. In den Begehungen wurden zusätzlich strukturierte Interviews zur Erfassung der Werte geführt. Tab. 3.2 fasst Ergebnisse der Artefakt-Erhebungen für die betrachteten Unternehmen zusammen.

Beide Unternehmen pflegen alle gesetzlich geforderten Dokumente und Vorgehensweisen für Arbeitssicherheit (gemäß Arbeitsschutzgesetz), wie Gefährdungsbeurteilungen, Unterweisungen, Begehungen, sowie bei Bedarf Unfalluntersuchungen; das Ziel Arbeitssicherheit ist in den Unternehmensleitlinien erfasst. In den Unternehmen ist der AGS formal verankert, die Umsetzung der formalen Strukturen in die Arbeitspraxen hat jedoch noch Potenziale. Bezogen auf die Sicherheitskultur haben die Unternehmen eher ein technikorientiertes, reaktives Verständnis von AGS; es zeigen sich deutliche Diskrepanzen im Umgang mit und in der gefühlten Sinnhaftigkeit von AGS je nach Bereich. In den Bürobereichen ist das Thema bekannt, aber wenig verinnerlicht; häufig ist der Bezug zum Thema Gesundheit eher individuell.

Um kulturrelevante Grundannahmen zu identifizieren, wurde eine Gap-Analyse [25] durchgeführt. Die in den Unternehmen der Praxispartner erhobenen Artefakte wurden als Abbilder gelebter Sicherheit - mit den Ergebnissen der Dokumentenanalyse, Interviews und Erhebungsworkshops, die Hinweise auf die gewünschte Sicherheitskultur liefern (Fokus: geäußerte Werte), verglichen. Für die Identifikation von Grundannahmen haben insbesondere „Brüche“ zwischen sichtbarer Kultur und geäußerten Werten hohe diagnostische Relevanz. Die Gap-Analyse erfolgte strukturiert anhand der vorab analytisch festgelegten Kulturindikatoren. Ergebnis der Gap-Analyse ist einerseits die Abbildung des Status der aktuellen Sicherheitskultur und ermöglicht anderseits die 
Tab. 3.2 Artefakte der Sicherheitskultur in den Unternehmen der Praxispartner

\begin{tabular}{l|l|l}
\hline Artefakte & Bauunternehmung & Komponentenherstellung \\
\hline $\begin{array}{l}\text { Sichtbarkeit } \\
\text { von Zielen }\end{array}$ & $\begin{array}{l}\text { Ziele in Dokumentation erfasst, im } \\
\text { Unternehmen wenig präsent bzw. nicht } \\
\text { sichtbar }\end{array}$ & $\begin{array}{l}\text { Ziele in Dokumentation verankert, } \\
\text { nicht durchgehend bekannt, teilweise } \\
\text { über Aushänge sichtbar }\end{array}$ \\
\hline $\begin{array}{l}\text { Nutzung der } \\
\text { destrumente AGS }\end{array}$ & $\begin{array}{l}\text { Alle rechtrelevanten Instrumente vor- } \\
\text { handen. Wenig Nutzung und Bekannt- } \\
\text { heit in der Belegschaft } \\
\text { Gefährdungsbeurteilung durch } \\
\text { externen Dienstleister, sehr formal, } \\
\text { wenig bekannt in Belegschaft } \\
\text { Unterweisungen finden statt, sehr } \\
\text { formale frontale Durchführung } \\
\text { Begehungen durch Sicherheits- } \\
\text { und Gesundheitsschutzkoordinator } \\
\text { (SiGeKo) auf Baustelle }\end{array}$ & $\begin{array}{l}\text { Alle rechtsrelevanten Instrumente } \\
\text { vorhanden. Nutzung im Arbeitsalltag } \\
\text { eingeschränkt } \\
\text { Gefährdungsbeurteilung durch } \\
\text { externen Dienstleister, wenig bekannt } \\
\text { in der Belegschaft } \\
\text { Unterweisungen werden durch- } \\
\text { geführt, sehr formale Durchführung } \\
\text { Begehungen durch externen Dienst- } \\
\text { leister ohne Einbindung von Mit- } \\
\text { arbeitenden }\end{array}$ \\
\hline $\begin{array}{l}\text { Organisation } \\
\text { Aufbau und }\end{array}$ & $\begin{array}{l}\text { Externe Betreuung, AMS System, } \\
\text { Pflichtenübertragungen liegen vor, } \\
\text { Verantwortlichkeiten sind nicht durch- } \\
\text { gängig bekannt }\end{array}$ & $\begin{array}{l}\text { Externe Betreuung für GB und ASA, } \\
\text { eigene Sicherheitsfachkraft } \\
\text { Verantwortlichkeiten über } \\
\text { Management Handbuch geregelt, aber } \\
\text { nicht durchgängig bekannt }\end{array}$ \\
\hline $\begin{array}{l}\text { Umgebung } \\
\text { und Zustände }\end{array}$ & $\begin{array}{l}\text { Zwei Welten: Büro vs. Baustelle } \\
\text { Büro: ordentlich, licht, auf } \\
\text { Kommunikation ausgerichtet } \\
\text { Baustelle: viel „,Durcheinander“, einige } \\
\text { unsichere Zustände }\end{array}$ & $\begin{array}{l}\text { Sauberkeit \& Ordnung nicht durch- } \\
\text { gängig } \\
\text { Teilweise unsichere Zustände }\end{array}$ \\
\hline $\begin{array}{l}\text { Aüro: Gesundheit als Thema (gesunder } \\
\text { Mittagstisch) } \\
\text { Baustelle: einige unsichere Hand- } \\
\text { lungen, Fremdfirmen werden anders } \\
\text { behandelt }\end{array}$ & $\begin{array}{l}\text { Diskrepanz zwischen Produktion und } \\
\text { Büro-/Verwaltungsbereichen }\end{array}$ \\
\hline
\end{tabular}

Ableitung konkreter Handlungsfelder für eine Kulturentwicklung. In den Erhebungen zeigte sich, dass insbesondere die kohärente und verständliche Darstellung der Themen des Arbeits- und Gesundheitsschutzes und der damit verbundenen Werten in Leitlinien und Unternehmenszielen anwendungsrelevant sind. Deutlich wurde auch, dass diese eine gute Basis für den Transformationsprozess hin zu Digitalisierung bieten und ihre Übertragung in allgemeingültige Regelungen anwendungsrelevant ist. In den Unternehmen der Praxispartner sind zwar die Inhalte des AGS in den Leitlinien auffindbar, diese sind aber im Unternehmen kaum bekannt und finden entsprechend im Arbeitsalltag wenig konsequente Umsetzung. Bezogen auf die Führung zeigt sich, dass die Verantwortlichkeiten und Zuständigkeiten im AGS in der Aufbauorganisation abgebildet sind, aber nicht durchgängig bei den Führungskräften bekannt sind. Auch der Wissensstand zu aktuellen Themen und Inhalten des AGS der Führungskräfte war nicht durchgängig 
Tab. 3.3 Ausprägung bezüglich der Kulturindikatoren

\begin{tabular}{l|l|l}
\hline Kulturindikator & Bauunternehmen & Komponentenfertigung \\
\hline Werte & $\begin{array}{l}\text { Verantwortung als schwieriges Thema } \\
\text { Vertrauen eingeschränkt } \\
\text { Hohe Verpflichtung bezüglich der } \\
\text { Fertigstellung des Bauprojekts }\end{array}$ & $\begin{array}{l}\text { Respektvolles Miteinander } \\
\text { Vertrauen untereinander }\end{array}$ \\
\hline Führung & $\begin{array}{l}\text { Konsequenz in der Umsetzung nicht } \\
\text { durchgängig } \\
\text { Vorbild eingeschränkt }\end{array}$ & $\begin{array}{l}\text { Konsequenz als Thema } \\
\text { Wenig Vorbildfunktion }\end{array}$ \\
\hline Kommunikation & $\begin{array}{l}\text { Fehlende Transparenz } \\
\text {,Rauer“ Umgangston }\end{array}$ & $\begin{array}{l}\text { Fehlende Klarheit/Transparenz } \\
\text { Offene Kommunikation }\end{array}$ \\
\hline Einbindung & $\begin{array}{l}\text { Starke Trennung zwischen Bereichen } \\
\text { der Planung und Ausführung }\end{array}$ & $\begin{array}{l}\text { Einbindung nicht flächendeckend } \\
\text { Abteilungsdenken }\end{array}$ \\
\hline Regelungen & $\begin{array}{l}\text { Regeln und Verantwortlichkeiten teil- } \\
\text { weise unbekannt viele Kompromisse }\end{array}$ & $\begin{array}{l}\text { Regeln vorhanden aber wenig } \\
\text { bekannt } \\
\text { Kompromisse bei Einhaltung }\end{array}$ \\
\hline
\end{tabular}

auf dem aktuellen Stand. In Tab. 3.3 sind die Ergebnisse der Gap-Analyse qualitativ aggregiert auf die Kulturindikatoren abgebildet.

Insgesamt wird deutlich, dass das Thema Arbeits- und Gesundheitsschutz bisher nicht kohärenter Teil der Unternehmenskultur im Sinne einer ganzheitlichen Präventionskultur ist und die Verbindung zu Themen der Digitalisierung wenig hergestellt ist.

Da Kultur als verhaltensbeeinflussendes Element in direkter Abhängigkeit von den Strukturen und Prozessen des Unternehmens steht, stellt sich die Frage nach der Nutzbarkeit der im Unternehmen etablierten Instrumenten des AGS, die eine die digitale Transformation stützen. Versteht man AGS systemisch mit dem Ziel, Arbeit menschengerecht zu gestalten, sollte geprüft werden, welche der auf Prävention ausgelegten Instrumente des Arbeitsschutzes im Sinne eines Managementsystems für die Transformation nutzbar gemacht werden können.

Um eine Implementierung der Instrumente in die digitale Arbeitswelt zu gewährleisten und deren Potenziale ausschöpfen zu können, wurden die AGS-Instrumente hinsichtlich ihrer Nutzbarkeit, Übertragbarkeit und Anwendbarkeit geprüft. Auswahlkriterien waren: Rechtssicherheit (gesetzlich gefordert), Systembezug (bezieht das gesamte Arbeitssystem ein), Prozesscharakter (gemäß PDCA-Zyklus), Empowerment-Potential (Einbezug der Beschäftigten, ermöglicht lernen). Ein wichtiges Auswahlkriterium war, dass KMU die Instrumente ohne Expertenwissen anwenden und ohne hohe Ressourcenbelastung (zeitlich und finanziell) umsetzen können. Das Ergebnis der Bewertung ist in Tab. 3.4 als Rangfolge dargestellt.

Fazit ist, dass drei Instrumente - Gefährdungsbeurteilung, Begehung und Unterweisung - ein hohes Nutzenpotential für eine Sicherheitskultur wie auch die Digitalisierung besitzen. Diese werden in den betrachteten Unternehmen genutzt - da dies gesetzlich gefordert wird -, allerdings wird ihr Potential bislang nicht ausgeschöpft. Die 
Tab. 3.4 Passung der AGS Instrumente zu Kriterien der digitalisierten Arbeitswelt

\begin{tabular}{l|l|l|l|l}
\hline Instrument & $\begin{array}{l}\text { Rechts- } \\
\text { Sicherheit }\end{array}$ & $\begin{array}{l}\text { System- } \\
\text { bezug }\end{array}$ & $\begin{array}{l}\text { Prozess- } \\
\text { charakter }\end{array}$ & $\begin{array}{l}\text { Empowerment- } \\
\text { Potential }\end{array}$ \\
\hline 1. Gefährdungsbeurteilung & ++ & ++ & ++ & ++ \\
\hline 2. Begehungen & + & + & ++ & ++ \\
\hline 3. Unterweisungen & + & + & ++ & ++ \\
\hline 4. Betriebsanweisungen & + & - & + & ++ \\
\hline 5. Erlaubnisscheinsystem & -- & ++ & ++ & ++ \\
\hline 6. Ereignis- / Unfallanalysen & ++ & ++ & -- & + \\
\hline 7. Audits & -- & ++ & ++ & -- \\
\hline 8. Meldung Beinahe-Unfälle & -- & -- & -- & ++ \\
\hline 9. Verbandbuch & - & -- & -- & + \\
\hline
\end{tabular}

(Legende: $++=$ sehr hoch; $+=$ gut; $-=$ eingeschränkt; $--=$ niedrig)

Ergebnisse zu verhaltensorientierten Nutzerpotenzialen (expliziter Wunsch nach Schulung und Weiterbildung) indizieren, dass insbesondere Unterweisungen geeignet sind, Themen der Digitalisierung bedarfsorientiert aufzugreifen, unternehmensspezifisch anzupassen und mitarbeiterorientiert umzusetzen.

Um eine präzise Abbildung der Sicherheitskultur zu ermöglichen, wurden zu den übergeordneten Kulturindikatoren generativ Subindikatoren gebildet. Zunächst wurden alle möglichen Faktoren aufgenommen, iterativ verdichtet und aggregiert. Die erste Iteration der Subindikatoren erfolgte auf Basis der Erhebungen und der Gap-Analyse mit dem Fokus auf Subindikatoren, die im direkten Bezug zum Arbeits- und Gesundheitsschutz stehen. In der zweiten Iteration wurden die Ergebnisse der Erhebungen zum Transformationsprozess (Abschn. 3.2.1) und Aspekten der Digitalisierung (Abschn. 3.2.2) einbezogen. Die Ergebnisse werden in Tab. 3.5 dargestellt.

Insgesamt erweist sich das Vorgehen als zielführend ist, da Indikatoren die Möglichkeit bieten, den aktuellen Stand der Sicherheitskultur 4.0 unternehmensspezifisch abzubilden und konkrete Ziele für den Transformationsprozess abzuleiten. Anhand der Kulturindikatoren lassen sich Handlungsfelder für Strategien und Maßnahmen eindeutig und unternehmensspezifisch bestimmen. Dies ist wichtig, da deutlich wurde, dass es im unternehmerischen Kontext nicht EINE Lösung bzw. Vorgehensweise gibt, sondern spezifische Voraussetzungen und Bedingungen berücksichtigt werden müssen, wie z. B. branchenspezifische Prozesse und Prozessabläufe und der Einfluss der Digitalisierung auf diese. Neben den organisatorisch-strukturellen Spezifika sind - wie in Abschn. 3.3.1.1 ausgeführt - personenbezogene Besonderheiten $\mathrm{zu}$ beachten, wie Alter, Geschlecht und Ausbildungsstand der Führungskräfte und Mitarbeitenden.

Basierend auf den Erhebungen und Ergebnissen zu Kulturindikatoren wurde ein Kriterienkatalog erstellt, der es erlaubt, unternehmensspezifische Besonderheiten zu beschreiben, zu erfassen und mit Handlungsfeldern im Unternehmenskontext zu 
Tab. 3.5 Kulturindikatoren und Subindikatoren einer Sicherheitskultur 4.0

\begin{tabular}{|c|c|}
\hline Kulturindikator & Subindikatoren Sicherheitskultur 4.0 \\
\hline Werte & $\begin{array}{l}\text { - Vertrauen (Verlässlichkeit) } \\
\text { - Respekt } \\
\text { - Wertschätzung }\end{array}$ \\
\hline Führung & $\begin{array}{l}\text { - Konsequenz („Kümmern“) } \\
\text { - Vorbild (Regelkonformität) }\end{array}$ \\
\hline Kommunikation & $\begin{array}{l}\text { - Transparenz } \\
\text { - Bereitstellung von Information } \\
\text { - Erhalt von Information (Durchgängigkeit über Schnittstellen) } \\
\text { - Informelle Kommunikation (Umgangston, Dialog, Rückmeldung) }\end{array}$ \\
\hline Einbindung & $\begin{array}{l}\text { - Partizipation und Mitsprache } \\
\text { - Identifikation mit Aufgaben und Arbeit } \\
\text { - Weiterbildung/Schulung }\end{array}$ \\
\hline Regelungen & $\begin{array}{l}\text { - Zuständigkeit/Verantwortlichkeiten } \\
\text { - Rolle der Regeln (Gültigkeit) }\end{array}$ \\
\hline
\end{tabular}

hinterlegen. Hierzu wurden die Kulturindikatoren und Subindikatoren mit den drei relevanten Umsetzungsebenen im Unternehmen verknüpft: 1) auf der organisationalen Ebene durch Einbindung der Subindikatoren in die strategische Planung und den strukturellen Aufbau, 2) auf der Gruppenebene durch Umsetzung der Subindikatoren in relevanten Gruppierungen (Abteilungen, Mitarbeitergruppen, Fachgruppen) und 3) auf der Ebene des Individuums durch Umsetzung der Subindikatoren auf individueller Ebene (vgl. Abb. 3.17).

Damit der Kriterienkatalog von KMU für ein Screening der Sicherheitskultur genutzt werden kann, wurden zu den Einzelaspekten Leitfragen formuliert, die neben der Beschreibung der aktuellen Situation den Dialog über Entwicklungspotenziale forcieren und die Ableitung von Maßnahmen für die Transformation unterstützen. Für die Konkretisierung von Inhalten wurden exemplarische Szenarien formuliert, die als Diskussionsgrundlage im Unternehmen genutzt werden können.

\subsubsection{Beitrag zu übergeordneten Zielen}

Die Digitalisierungsmaßnahmen tragen dazu bei, Arbeitsinnovationen wie die papierlose Fertigung im Unternehmen zu etablieren und darauf aufbauend Mitarbeitern rollen-, aufgaben- und kontextspezifische Sicherheitsinformationen zur Verfügung zu stellen, um die gesundheitsförderliche Arbeitssystemgestaltung im digitalen Zeitalter zu gewährleisten. Derzeit werden weitere Kommunikations- und Empowermentmaßnahmen bei beiden Praxispartnern erprobt, ihre Auswirkungen werden anschließend evaluiert. Die Ergebnisse werden in weiteren Publikationen (beispielsweise [8]) veröffentlicht. 


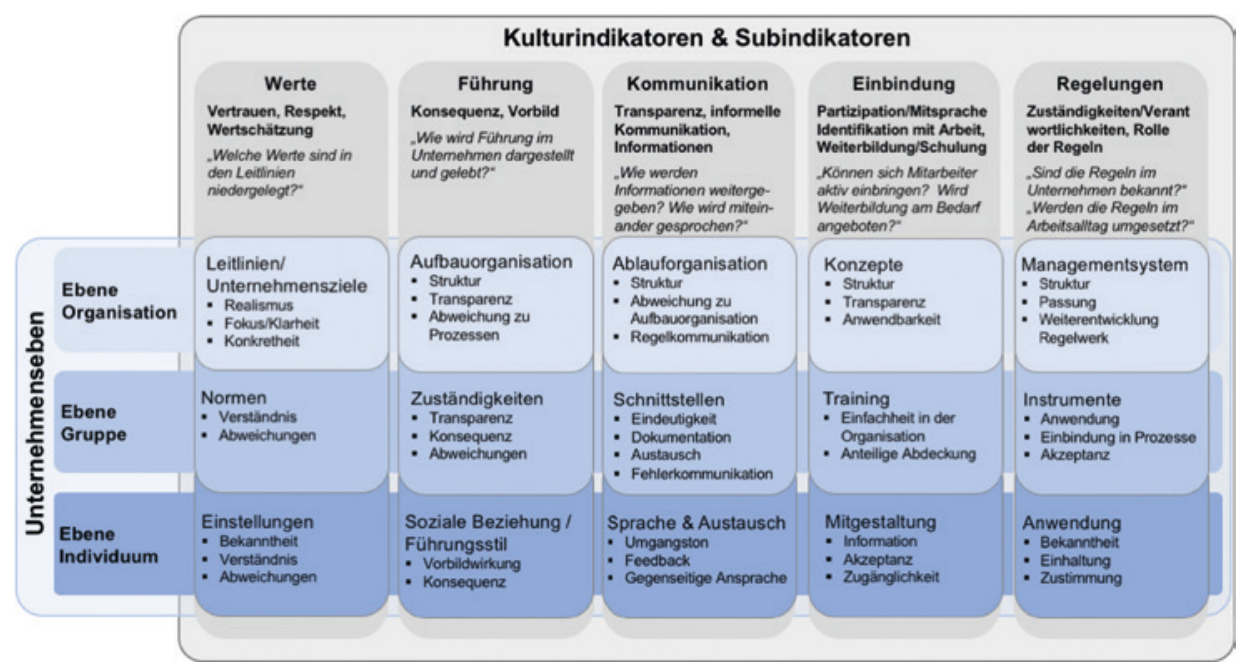

Abb. 3.17 Kriterienkatalog Sicherheitskultur 4.0

\subsection{Lessons learned}

Im Folgenden werden kritische Punkte und Erfolgsfaktoren für die Entwicklung und Etablierung einer Sicherheitskultur als Transformationsansatz für Industrie 4.0 in kleinen und mittleren Unternehmen zusammengefasst.

- Digitalisierung ist immer Chance und Risiko zugleich: Digitale Technologien lösen bestehende Probleme, ziehen jedoch Risiken nach sich, deren konkrete Auswirkungen schwer vorherzusagen sind. Die Umsetzung einer Sicherheitskultur 4.0 bedarf daher neben der sorgfältigen Entwicklung und Auswahl passender Strategien und Maßnahmen in Unternehmen dem kontinuierlichen Monitoring hinsichtlich der Frage, ob sich neue digitale Technologien negativ auf die Sicherheit im Arbeitskontext auswirken.

- Sicherheitskultur braucht Profil: Herausforderrungen in der Entwicklung des Transformationsansatzes stellten insbesondere der unterschiedliche Grad der Digitalisierung sowie der andersartige Umgang mit der Arbeitssicherheit innerhalb verschiedener industrieller Branchen dar. Erst durch den Einsatz von Kulturindikatoren und dem darauf bezogenen Kriterienkatalog können der aktuellen Status der unternehmensinternen Sicherheitskultur abgebildet, konkrete Handlungsfelder für Interventionen identifiziert und über Leitfragen eine diskursive, von allen Mitgliedern des Unternehmens getragenen Kulturentwicklung ermöglicht werden.

- Das Schneeballprinzip nutzen: Eine Sicherheitskultur 4.0 kann nicht "angeordnet" werden, sondern nur in einem Organisationsentwicklungsprozess implizit durch 
Sozialisation, Beteiligung, Kommunikation und Führung gestützt durch explizite Regeln und Strukturen vermittelt, die auf den Instrumenten des AGS sowie auf Transformations-, Kommunikations- und Empowerment-Maßnahmen basieren. In diesem Sinne muss eine Sicherheitskultur 4.0 ,top-down' vom Management initiiert und begleitet sowie ,bottom-up“ durch Promotoren auf Mitarbeiterebene etabliert werden, die andere Mitarbeiter von den Vorteilen einer sicherheits-fördernden Digitalisierung überzeugen.

- Sicherheitskultur 4.0 ist stetiger Wandel: Eine Sicherheitskultur 4.0 stellt einen Paradigmenwechsel hinsichtlich der Arbeit in sich wandelnden Kontexten dar. Viele etablierte Strukturen und Verhaltensweisen müssen aufgebrochen und durch neue ersetzt werden. Eine Sicherheitskultur 4.0 ist geprägt von einem kontinuierlichen Wandel, um neuen technologischen Entwicklungen und damit verbundenen Risiken begegnen zu können.

- Sicherheitskultur heißt Prävention: Ziel sollte eine Präventionskultur sein, die im Sinne eines systemischen Arbeitsschutzverständnisses Arbeit in all ihren Facetten menschengerecht gestaltet und nicht retrospektiv formelle Verhaltensrichtlinien und informelle Praxen an neu auftretende Risiken anpasst. Eine solche Kultur der Prävention geht davon aus, dass Sicherheit und Gesundheit Werte für alle Menschen, jede Organisation und die Gesellschaft sind und präventives Handeln lohnend und sinnstiftend ist (vgl. [19]).

\section{Literatur}

1. Baethge C, Boberach Kantar M. (2018) Zukunft der Arbeit in deutschen KMU. Werkstattbericht. https://www.bertelsmann-stiftung.de/fileadmin/files/BSt/Publikationen/ GrauePublikationen/Zukunft_der_Arbeit_in_deutschen_KMU_Werkstattbericht.pdf. Zugegriffen: 27. August 2019

2. Bollmann U, Lee YJ, Seo Y, Paridon H, Kohstall T, Hessenmöller AM, Bochmann C (2018 accepted). Leading Indicators for a Culture of Prevention. Prevention Science, Special Issue on international developments of a culture of prevention, New York: Springer

3. Borg A, Wilde J, Dziadus D, Pieper C, Boczek B, Bauer M (2011) PARSAG - Partizipatives, systemisches Arbeits- und Gesundheitsschutzmanagement. Abschlussbericht. Bexbacher/ Aachener Beiträge für Consulting, Business und Management, Band 5

4. Digmayer C, Jakobs EM (2014) Corporate Lifelong Learning 2.0: Design of Knowledge Management Systems with Social Media Functions as Learning Tools. Proceedings of the IEEE International Professional Communication Conference 2014, doi: https://doi. org/10.1109/IPCC.2014.7020352

5. Digmayer C, Jakobs EM (2018) Employee Empowerment in the Context of domain-specific Risks in Industry 4.0. Proceedings of the International Professional Communication Conference 2018. doi: https://doi.org/10.1109/ProComm.2018.00034

6. Digmayer C, Jakobs EM (2019) Let's put the V in smart factory: Empowering employees to shape a safety culture for Industry 4.0. COMA 2019 - International Conference on Competitive Manufacturing. https://scholar.sun.ac.za/handle/10019.1/105429. Zugegriffen am 27. August 2019 
7. Digmayer C, Jakobs EM (2019) Developing Safety Cultures for Industry 4.0. New Challenges for Professional Communication. Proceedings of the International Professional Communication Conference 2019. doi: https://doi.org/10.1109/ProComm.2019.00045

8. Digmayer C, Jakobs EM (In Vorbereitung) Using video tutorials as mean to foster employee empowerment and a shared understanding of safety in Industry 4.0.

9. Edwards J et al (2013) Returning to the roots of culture: a review and re-conceptualisation of safety culture. Safety Science 55:70-80.

10. Elke G et al (2015) Arbeitsschutz und betriebliche Gesundheitsförderung - vergleichende Analyse der Prädiktoren und Moderatoren guter Praxis. Bundesanstalt für Arbeitsschutz und Arbeitsmedizin. https://d-nb.info/1071866893/34. Zugegriffen am 27. August 2019

11. Flin R et al (2000) Measuring safety climate: Identifying the common features. Safety Science 34:177-192 the common features. Safety Science 34:177-192

12. Gudergan G., Feige B. und Krechting D. (2017). Ordnungsrahmen für den Prozess der BusinessTransformation, in: Wolfgang Pollety, Andreas Blaeser-Benfer (Hg.), Digitalisierung, Betriebliche Handlungsfelder der Unternehmensentwicklung, Frankfurter Allgemeine Buch.

13. Guldenmund F.W (2000) The nature of safety culture: a review of theory and research. In: Safety Science 34 (1-3):215-257

14. Guldenmund FW (2007) The use of questionnaires in safety culture research - an evaluation. Safety Science 45(6):723-743

15. Guldenmund, FW (2016) Organizational Safety Culture. In: Clarke S et al (Hrsg) The Wiley Blackwell handbook of the psychology of occupational safety and workplace health. New Jersey, Wiley Blackwell Publishing Ltd., S 437-458

16. Hildebrandt, J., Kluge, J., \& Ziefle, M. (2019) Work in Progress: Barriers and Concerns of Elderly Workers Towards the Digital Transformation of Work. (158-169) doi: https://doi. org/10.1007/978-3-030-22012-9_12

17. Jakobs EM et al (2011) Industrielle Prozessmodellierung als kommunikativer Prozess. Eine Typologie zentraler Probleme. In: Gesprächsforschung 12:223-264

18. Kluge J, Hildebrandt J, Ziefle M (2019) The Golden Age of Silver Workers? doi:https://doi. org/10.1007/978-3-030-22015-0_40

19. Kommmitmensch (DGUV) URL: https://www.kommmitmensch.de/die-kampagne/. Zugegriffen am 27. August 2019

20. Kooij D et al (2010) The influence of age on the associations between HR practices and both affective commitment and job satisfaction: A meta-analysis. Journal of Organizational Behavior 31(8):1111-11361111-1136

21. Kooij D et al (2013) How the impact of HR practices on employee well-being and performance changes with age. Human Resource Management Journal 23(1):18-35

22. Niederdrenk R, Seemann R (2018) Baubranche aktuell: Wachstum 2020 - Digitalisierung und BIM. https://www.pwc.de/de/industrielle-produktion/baubranche-aktuell-wachstum-2020maerz-2018.pdf. Zugegriffen am 27. August 2019

23. Nink M (2014) Engagement Index: Die neuesten Daten und Erkenntnisse aus 13 Jahren Gallup-Studie. Redline Wirtschaft, München

24. Ruppel, C. P., \& Harrington, S. J. (2000). The relationship of communication, ethical work climate, and trust to commitment and innovation. Journal of business Ethics, 25(4), 313-328. doi: 10.1023/A:1006290432594

25. Schein EH (2010) Organizational culture and leadership (4th edition). Jossey-Bass, San Francisco

26. Schöbel M et al (2017) Digging deeper! Insights from a multi-method assessment of safety culture in nuclear power plants based on Schein's culture model. Safety Science 95:38-493849 
27. Schuh G et al (2017) Industrie 4.0 Maturity Index: Die digitale Transformation von Unternehmen gestalten. Herbert Utz Verlag, München

28. Spreitzer GM (1995) Psychological empowerment in the workplace: Dimensions, measurement, and validation. Academy of Management Journal 38(5):1442-1465

29. Strom U, Ulich E (1997) Unternehmen arbeitspsychologisch bewerten. Ein Mehr-Ebenen-Ansatz unter besonderer Berücksichtigung von Mensch, Technik und Organisation. Vdf Hochschulverlag, Zürich

30. Tauchert J, Thiessen T (2018) Digitalisierung der mittelständischen Bauwirtschaft in Deutschland. Statusevaluation und Handlungsempfehlungen. https://kommunikation-mittelstand. digital/content/uploads/2018/10/Status-Quo_Digitalisierung_Bauwirtschaft.pdf. Zugegriffen am 27. August 2019

Open Access Dieses Kapitel wird unter der Creative Commons Namensnennung 4.0 International Lizenz (http://creativecommons.org/licenses/by/4.0/deed.de) veröffentlicht, welche die Nutzung, Vervielfältigung, Bearbeitung, Verbreitung und Wiedergabe in jeglichem Medium und Format erlaubt, sofern Sie den/die ursprünglichen Autor(en) und die Quelle ordnungsgemäß nennen, einen Link zur Creative Commons Lizenz beifügen und angeben, ob Änderungen vorgenommen wurden.

Die in diesem Kapitel enthaltenen Bilder und sonstiges Drittmaterial unterliegen ebenfalls der genannten Creative Commons Lizenz, sofern sich aus der Abbildungslegende nichts anderes ergibt. Sofern das betreffende Material nicht unter der genannten Creative Commons Lizenz steht und die betreffende Handlung nicht nach gesetzlichen Vorschriften erlaubt ist, ist für die oben aufgeführten Weiterverwendungen des Materials die Einwilligung des jeweiligen Rechteinhabers einzuholen.

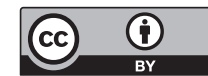

